INRA Prod. Anim.,

2012, 25 (3), 277-290

\title{
Aptitude des chèvres hautes productrices de lait à valoriser les prairies temporaires au pâturage
}

\author{
Y. LEFRILEUX ${ }^{1}$, P. MORAND-FEHR 2,3, A. POMMARET 4 \\ ${ }^{1}$ Institut de l'Elevage Station Expérimentale Caprine du Pradel, F-07170 Mirabel, France \\ 2 INRA, UMR791 Modélisation Systémique Appliquée aux ruminants, \\ 16 rue Claude Bernard, F-75005 Paris, France \\ ${ }^{3}$ AgroParisTech, UMR Modélisation Systémique Appliquée aux ruminants, \\ 16 rue Claude Bernard, F-75005 Paris, France \\ ${ }^{4}$ Station Expérimentale Caprine du Pradel, EPLEFPA, F-07170 Mirabel, France \\ Courriel : morand@agroparistech.fr
}

En France, en système de production intensive, la production de lait de chèvre se situe en majorité entre 800 et $1100 \mathrm{~kg} /$ chèvre/an. Dans ces systèmes le pâturage est assez peu utilisé en particulier parce que la chèvre est supposée mal l'utiliser. Les travaux réalisés au Domaine du Pradel en Ardèche ont précisé les conditions - type de pâturage, mise à l'herbe, complémentation - permettant de conduire au pâturage des chèvres à fort niveau de production laitière.

La chèvre laitière, haute productrice, qui produit $800 \mathrm{~kg}$ ou plus de lait par an dans les conditions de l'élevage caprin en France, a souvent été considérée comme une médiocre utilisatrice du pâturage cultivé en raison de la proportion importante de refus qu'elle laisse sur la prairie (Morand-Fehr 1981, Simiane et al 1981). C'est la raison pour laquelle il existe peu de travaux sur les systèmes d'alimentation à base de pâturage utilisé par la chèvre laitière dans des conditions intensives à la différence de la vache laitière (Peyraud et al 1995, Delaby et al 2003) et de la brebis laitière (Sitzia et al 1997, Ligios et al 2002). Chez la chèvre laitière, Jagusch et al (1981) ont obtenu dans des conditions néo-zélandaises des niveaux de production journalière de $2,6 \mathrm{~kg}$ de lait chez des chèvres en pâturage tournant, recevant seulement $200 \mathrm{~g}$ de concentré par jour et Soryal et al (2004) en Inde en conditions tropicales, $2,9 \mathrm{~kg}$ de lait avec $900 \mathrm{~g}$ de concentré par jour. Masson et Simiane (1981) ont aussi observé en France que des chèvres alpines peuvent consommer des quantités moyennes d'herbe de 70 et $50 \mathrm{~g}$ de MS par $\mathrm{kg}$ $\mathrm{P} 0,75$ par jour, respectivement sur prairie de légumineuses et de graminées en pâturage rationné et que dans ces conditions, elles produisent en moyenne 2,4 kg de lait par jour. Min et al (2005), plus récemment aux Etats-Unis, ont obtenu des performances plus élevées, de 2,0 à 3,8 kg de lait par jour, avec des chèvres alpines à potentiel laitier élevé pâturant des prairies temporaires d'herbe tropicale et tempérée et recevant peu ou pas d'aliments concentrés.

Au cours des dernières années, plusieurs facteurs ont incité certains éleveurs de chèvres laitières à revenir à des systèmes d'alimentation à base de pâture. Pour soutenir l'élevage caprin dans les pays industrialisés, l'introduction de conduites alimentaires utilisant l'espace en abandonnant des méthodes de confinement en chèvrerie, permet de maintenir une image plus proche de ce que cet élevage représente pour le consommateur et de répondre à la demande sociétale de ces pays. De plus, les cahiers des charges des AOP incitent fortement les producteurs à pratiquer le pâturage. En France cette orientation s'inscrit dans un contexte où les chèvres, grâce aux programmes d'amélioration génétique, sont maintenant capables de produire plus de $1000 \mathrm{~kg}$ de lait par an (Barillet et al 2004). En outre, les consommateurs de fromages de chèvre deviennent de plus en plus nombreux et exigent que ces fromages présentent une pâte fine et onctueuse et un goût de chèvre caractéristique, sans être trop marqué et sans mauvais goût. Les consommateurs estiment que ce type de fromage a une vocation légitime à être fabriqué plutôt avec du lait de chèvre recevant une alimentation considérée comme «naturelle», notamment à base de pâturage, qu'avec des rations très riches en aliments concentrés fabriqués industriellement.

La station expérimentale caprine du Pradel faisant partie du Pôle d'Expérimentation et de Progrès Caprin (PEP Caprin) de la région Rhône-Alpes a consacré la majorité de ses travaux depuis près de 15 ans, à l'utilisation du pâturage cultivé par la chèvre haute productrice de lait. Elle a obtenu des résultats originaux, en particulier sur la conduite des chèvres au pâturage, le type de pâturage, la mise à l'herbe et la stratégie de complémentation à adopter. En conséquence, nous rapportons dans cet article, les principaux résultats de ces travaux dont une partie a déjà été publiée dans divers articles en français et en anglais (Van Quackebeke et al 1996a et b, 1997 ; Lefrileux et al 2000, 2005, 2008) afin de montrer qu'il est possible d'obtenir un niveau de production élevée de $1000 \mathrm{~kg}$ à $1100 \mathrm{~kg}$ de lait par chèvre et par an avec du pâturage comme principal apport fourrager du régime. Nous présentons principalement les performances laitières (production de lait, taux butyreux et protéique) sans aborder les aspects de 
composition en acide gras et de teneurs en vitamines et micronutriments du lait de chèvre puisqu'ils ont déjà été traités dans un précédent article (MorandFehr et al 2007) et plus généralement dans les articles de Chilliard et al (2003, 2007) et de Fedele et al (2007). L'objectif de ces travaux était donc de mieux définir les conditions d'utilisation de l'herbe pâturée par la chèvre laitière produisant plus de $800 \mathrm{~kg}$ de lait par an et les systèmes d'alimentation où l'herbe pâturée représente la majeure partie de l'énergie de la ration ingérée quotidiennement dans les conditions de l'élevage caprin en France.

\section{1 / Conduite du troupeau expérimental du Pradel}

La station expérimentale du Pradel est située dans le département de l'Ardèche, au Sud-est de la France (latitude $44^{\circ} 35$, longitude $4^{\circ} 30$ ), sur les collines du Vivarais (altitude $280 \mathrm{~m}$ ). Elle bénéficie d'un climat méditerranéen humide. Le relief de l'exploitation est caractérisé par de faibles pentes où sont situées les pâtures. Les sols sont de type argileux à argilo-limoneux (30 à 50\% d'argile) avec une alternance de strates marneuses et marno-calcaires. Le $\mathrm{pH}$ moyen des sols est de 7,80 avec une forte hétérogénéité $(6,90$ à 8,40$)$ liée à la présence d'un soubassement érodé recouvert de cailloutis basaltiques et partiellement d'alluvions fines.

La température moyenne annuelle est de $13,1^{\circ} \mathrm{C}$ avec un minimum moyen en Janvier de $1,1^{\circ} \mathrm{C}$ et un maximum moyen en Juillet de $29,3^{\circ} \mathrm{C}$. Le printemps est doux et précoce, l'été très chaud, l'automne doux et humide et l'hiver assez froid. La pluviométrie est élevée et atteint en moyenne $1050 \mathrm{~mm}$ par an. La répartition des pluies est irrégulière avec 2 pics, au printemps et surtout à l'automne. L'excès d'eau en automne rend difficile les travaux culturaux sur les pâtures et en particulier l'implantation de légumineuses. Le démarrage de la végétation a lieu en général début Mars. Le déficit hydrique en été est généralement important et stoppe la pousse de l'herbe pendant 2 à 3 mois. La repousse de l'herbe apparaît au cours $\mathrm{du}$ mois de Septembre et se poursuit jusqu'en Novembre.

Le troupeau expérimental comprend environ 120 chèvres de race alpine provenant de troupeaux inscrits au contrôle laitier et au livre généalogique de la race alpine. Leur poids moyen adulte varie entre 60 et $70 \mathrm{~kg}$ en fonction de leur stade physiologique. La production laitière moyenne est de $1100 \mathrm{~kg}$ par an, le taux butyreux de $38 \mathrm{~g} / \mathrm{kg}$ de lait et le taux protéique de $32,7 \mathrm{~g} / \mathrm{kg}$. La durée moyenne de lactation est d'environ 290 jours. Depuis 15 ans, l'utilisation de boucs améliorateurs et de l'insémination artificielle a permis d'améliorer sensiblement la production moyenne de lait du troupeau ainsi que sa composition. La période de reproduction se situe en Août avec des retours de chaleur en Septembre de telle manière que les mises bas démarrent au $1^{\text {er }}$ Janvier et se terminent juste avant le début de la mise à l'herbe vers le $1^{\text {er }}$ Mars.

A la chèvrerie, les fourrages sont distribués dans une auge pour chaque lot. Tous les fourrages distribués sont pesés chaque jour pour chaque lot et les refus 3 à 4 fois par semaine. Les aliments concentrés et les fourrages déshydratés sont distribués à l'aide d'un distributeur automatique d'aliment concentré; ce qui permet de pouvoir faire varier la nature et la quantité d'aliments complémentaires apportés à chaque chèvre laitière selon l'évolution de ses besoins alimentaires et selon les protocoles expérimentaux. Les chèvres ne sont pas abreuvées au pâturage mais seulement à la chèvrerie.

L'exploitation caprine de la station s'étend sur 20 ha de pâturage cultivé (14 ha de graminées et 6 ha de luzerne). La surface en luzerne permet d'allonger le pâturage en période estivale et celle en graminées permet des mises à l'herbe précoces au printemps $(5,2$ ha de ray-grass hybride, 5,2 ha de dactyle, et 3,6 ha de fétuque en moyenne). Les parcelles de luzerne reçoivent en moyenne 80 et 140 unités de $\mathrm{P}$ et $\mathrm{K}$ respectivement, et celles de graminées 40, 100 et 140 unités de N, P, K (engrais de fond $\mathrm{P}$ et $\mathrm{K}$ en fin d'hiver, apport azoté en deux passages, avant la mise à l'herbe et avant le 15 Mai et, si nécessaire, au redémarrage de la végétation après l'été). Par ailleurs, les refus sur les pâtures sont fauchés et broyés quand cela est nécessaire (en moyenne autour d'une fois par an) selon le programme d'utilisation des prairies.

Le pâturage est effectué sur des parcelles de 1 à 2 ha qui peuvent être subdivisées en fonction de l'objectif des expériences. La durée moyenne de présence des animaux sur les parcelles est d'environ 6 à 7 heures par jour en début et en fin de saison et de 10 à 12 heures pendant les périodes de fortes disponibilités fourragères. Dans ce cas, les animaux sont remis au pâturage après la traite du soir jusqu'à la tombée de la nuit. En moyenne, les animaux sont présents sur le pâturage pendant 230 jours par an (150 au printemps et 80 à l'automne) avec deux périodes d'interruption l'été de 35 jours environ du 20 Juillet au $1^{\text {er }}$ Septembre et de 100 jours en hiver de fin Novembre aux premiers jours de Mars.

\section{2 / Méthodes utilisées}

Au cours de toutes ces expériences, les performances laitières (quantité de lait, taux butyreux et protéique) étaient contrôlées individuellement un jour par semaine. Les chèvres étaient pesées une fois par mois. Leur Note d'Etat Corporel (NEC) était évaluée un jour par mois à la même heure par la méthode d'Hervieu et Morand-Fehr (1999) basée sur des palpations. Le fourrage distribué et les refus étaient pesés pour chaque lot. Enfin, les lots expérimentaux étaient constitués de façon équilibrée sur les critères suivants par ordre d'importance : production laitière, composition du lait, âge, date de mise bas, NEC, poids vif.

Les principaux éléments des protocoles des 9 expériences synthétisées dans cet article (thème de l'expérience, durée, nombre de lots, nombre de chèvres par lot, traitements et rations des différents lots de chèvres) ont été résumés dans le tableau 1 afin de disposer d'une base à laquelle le lecteur peut se rapporter, en particulier pour les expériences qui ont plusieurs objectifs et qui sont évoquées dans différentes parties de cet article.

\section{3 / Comparaison entre une alimentation au pâturage et une alimentation en chè- vrerie (expériences 1 et 2)}

Pour évaluer une conduite alimentaire au pâturage par rapport à une conduite en confinement où les chèvres restent toute la journée en chèvrerie et qui est encore une technique fréquente en France, deux expériences ont été réalisées à la station du Pradel. L'une a duré trois années et l'autre 100 jours.

\section{1 / En pâturage tournant (expérience 1)}

Cette expérience déjà décrite par Van Quackebeke et al (1996a) s'est déroulée au cours des 3 premières années qui suivirent la constitution du troupeau sur 2 surfaces mitoyennes d'environ 10 ha de valeur agronomique très comparable. Le même système fourrager y a été mis en place : $2 / 3$ de légumineuses $(90 \%$ de luzerne et $10 \%$ de sainfoin) et $1 / 3$ de graminées (fétuque et ray-grass anglais).

A partir de 102 chèvres, la première année et de 120 chèvres, les $2^{\text {ème }}$ et $3^{\text {ème }}$ 
Tableau 1. Objectifs et dispositifs expérimentaux des 9 expériences réalisées avec des chèvres laitières au pâturage au domaine du Pradel.

\begin{tabular}{|c|c|c|c|c|c|c|}
\hline $\begin{array}{l}N^{\circ} \text { de } \\
\text { l'exp. }\end{array}$ & Thème & Durée & $\begin{array}{l}\text { Nb.de } \\
\text { lots }\end{array}$ & $\begin{array}{l}\text { Nb. de } \\
\text { Chèvres } \\
\text { par lot }\end{array}$ & Traitements & Rations \\
\hline 1 & $\begin{array}{c}\text { Comparaison des systèmes } \\
\text { d'alimentation en chèvrerie } \\
\text { et au pâturage }\end{array}$ & $\begin{array}{c}3 \\
\text { ans }\end{array}$ & 2 & $50-60$ & $\begin{array}{c}\mathrm{H}: \text { Chèvrerie } \\
\mathrm{P}: \text { Pâturage tournant }\end{array}$ & $\begin{array}{c}\mathrm{H}: \text { Foin, } \mathrm{P}: \text { Pâturage } \\
\text { Pour les } 2 \text { lots : } \\
\text { Conc. : } 0,7-0,8 \mathrm{~kg} / \mathrm{j} \\
\text { Luz. Des : } 0,15-0,35 \mathrm{~kg} / \mathrm{j} .\end{array}$ \\
\hline 2 & Idem expérience 1 & $\begin{array}{c}100 \\
\text { jours }\end{array}$ & 2 & 51 & $\begin{array}{c}\text { Idem } \\
\mathrm{P}: \text { Pâturage continu }\end{array}$ & Idem expérience 1 \\
\hline 3 & $\begin{array}{l}\text { Comparaison } \\
\text { du pâturage tournant } \\
\text { et du pâturage continu }\end{array}$ & $\begin{array}{c}2 \\
\text { ans }\end{array}$ & 2 & 60 & $\begin{array}{c}\mathrm{T}: \text { Tournant } \\
\text { sur } 13 \text { parcelles } \\
\mathrm{C}: \text { Continu } \\
\text { sur } 3 \text { parcelles }\end{array}$ & $\begin{array}{l}\text { Pâturage tournant } \\
\text { ou continu } \\
+0,8 \mathrm{~kg} \text { concentré }\end{array}$ \\
\hline 4 & $\begin{array}{c}\text { Comparaison d'une mise } \\
\text { à l'herbe précoce vs tardive et } \\
\text { d'un concentré riche } \\
\text { en PDIA vs pauvre en PDIA }\end{array}$ & $\begin{array}{c}120 \\
\text { jours }\end{array}$ & 4 & 30 & $\begin{array}{c}\mathrm{P}: \text { MAH le } 1^{\mathrm{er}} \text { Mars } \\
\mathrm{T}: \text { MAH le } 31 \text { Mars } \\
\text { Sous lots PHP et THP : } \\
\text { Conc. riche en PDIA } \\
\text { Sous lots PBP et TBP : } \\
\text { Conc. pauvre en PDIA }\end{array}$ & $\begin{array}{l}\text { Pâturage se substituant } \\
\text { au foin }+0,8 \mathrm{~kg} / \mathrm{j} \\
\text { de concentré riche } \\
\text { ou pauvre en PDIA }\end{array}$ \\
\hline 5 & $\begin{array}{l}\text { Effet du niveau d'apport } \\
\text { en concentré au pâturage }\end{array}$ & $\begin{array}{l}84 \\
\text { jours }\end{array}$ & 5 & 24 & $\begin{array}{l}\text { A : } 0,26 \mathrm{~kg} / \mathrm{j} \text { Conc. } \\
\text { B : } 0,55 \mathrm{~kg} / \mathrm{j} \text { Conc. } \\
\text { C : } 0,80 \mathrm{~kg} / \mathrm{j} \text { Conc. } \\
\text { C : } 1,05 \mathrm{~kg} / \mathrm{j} \text { Conc. } \\
\text { E : } 1,30 \mathrm{~kg} / \mathrm{j} \text { Conc. }\end{array}$ & $\begin{array}{l}\text { Pâturage continu + apport } \\
\text { de concentré variant } \\
\text { de } 0,26 \text { à } 1,30 \mathrm{~kg} / \mathrm{j} \\
\text { selon les lots. }\end{array}$ \\
\hline 6 & $\begin{array}{l}\text { Effet du niveau d'apport } \\
\text { en concentré et de la nature } \\
\text { de la source énergétique } \\
\text { du concentré. }\end{array}$ & $\begin{array}{l}84 \\
\text { jours }\end{array}$ & 4 & 30 & $\begin{array}{c}\mathrm{A} 0,65: 0,65 \mathrm{~kg} / \mathrm{j} \text { Conc. } \\
\text { riche en amidon (A) } \\
\mathrm{A} 1,30: 1,30 \mathrm{~kg} / \mathrm{j} \text { Conc. } \mathrm{A} \\
\mathrm{F} 0,65: 0,65 \mathrm{~kg} / \mathrm{j} \text { Conc. } \\
\text { riche en fibres (F) } \\
\mathrm{F} 1,30: 1,30 \mathrm{~kg} / \mathrm{j} \text { Conc. } \mathrm{F}\end{array}$ & $\begin{array}{l}\text { Pâturage continu } \\
+0,65 \text { ou } 1,30 \mathrm{~kg} / \mathrm{j} \\
\text { de concentré riche } \\
\text { en amidon ou en fibre }\end{array}$ \\
\hline 7 & $\begin{array}{c}\text { Effet du niveau d'apport } \\
\text { de concentré au pâturage }\end{array}$ & $\begin{array}{l}100 \\
\text { jours }\end{array}$ & 2 & 17 & $\begin{array}{l}\mathrm{B}: 0,5 \mathrm{~kg} / \mathrm{j} \text { Conc. } \\
\mathrm{H}: 0,8 \mathrm{~kg} / \mathrm{j} \text { Conc. }\end{array}$ & $\begin{array}{l}\text { Pâturage continu }+0,5 \\
\text { ou } 0,8 \mathrm{~kg} / \mathrm{j} \text { de concentré }\end{array}$ \\
\hline 8 & $\begin{array}{c}\text { Comparaison de } 2 \text { niveaux } \\
\text { d'apport au pâturage } \\
\text { de maïs ou de concentré } \\
\text { du commerce }\end{array}$ & $\begin{array}{c}125 \\
\text { jours }\end{array}$ & 4 & 27 & $\begin{array}{l}\mathrm{HM}: 1 \mathrm{~kg} / \mathrm{j} \text { Maïs } \\
\mathrm{HC}: 1 \mathrm{~kg} / \mathrm{j} \text { Conc. } \\
\mathrm{BM}: 0.5 \mathrm{~kg} / \mathrm{j} \text { Maïs } \\
\mathrm{BC}: 0,5 \mathrm{~kg} / \mathrm{j} \text { Conc. }\end{array}$ & $\begin{array}{c}\text { Pâturage continu } \\
+0,5 \mathrm{~kg} / \mathrm{j} \text { ou } 1 \mathrm{~kg} / \mathrm{j} \\
\text { de maïs ou de concentré }\end{array}$ \\
\hline 9 & $\begin{array}{l}\text { Comparaison d'un apport } \\
\text { d'orge ou de concentré } \\
\text { du commerce au pâturage }\end{array}$ & $\begin{array}{l}100 \\
\text { jours }\end{array}$ & 2 & 17 & $\begin{array}{l}O: 0,8 \mathrm{~kg} / \mathrm{j} \text { Orge } \\
\mathrm{C}: 0,8 \mathrm{~kg} / \mathrm{j} \text { Conc. }\end{array}$ & $\begin{array}{l}\text { Pâturage continu }+0,8 \mathrm{~kg} / \mathrm{j} \\
\text { d'orge ou de concentré }\end{array}$ \\
\hline
\end{tabular}

Conc $=$ Aliment Concentré $;$ Luz. Des. = Luzerne Déshydratée $; \mathrm{MAH}=$ Mise à l'Herbe $; \mathrm{A}=\mathrm{Amidon} ; \mathrm{F}=\mathrm{Fibre}$.

années, deux lots de 50 puis de 60 chèvres, équilibrés en production et composition du lait, en poids vif et état corporel ont été constitués et répartis sur chacune des 2 surfaces fourragères. Les chèvres du lot $\mathrm{H}$ ou du lot $\mathrm{P}$ la première année, restent dans le même lot l'année suivante et les chèvres primipares sont introduites dans les lots de façon aléatoire. Le lot $\mathrm{H}$ était intégralement alimenté en chèvrerie à partir de foins récoltés et le lot $\mathrm{P}$ était conduit en pâturage tournant. Le lot $\mathrm{P}$ recevait du foin en complément du pâturage lorsque celui-ci était insuffisant, en particulier en été et en hiver ou bien pendant les jours où les chèvres ne pouvaient pas sortir (pluie, tempête). Ce foin provenait des excédents d'herbe récoltés au printemps sur les surfaces que le lot $\mathrm{P}$ pâturait.

Les apports complémentaires journaliers d'aliments concentrés et de luzerne déshydratée étaient identiques dans les 2 lots pendant les périodes où les animaux étaient conduits en chèvrerie. Pour le lot $\mathrm{P}$, les apports de fourrages déshydratés ont été progressivement diminués pendant les périodes de transition à l'herbe. La luzerne déshydratée était distribuée en quantité variable de 0 à $1 \mathrm{~kg}$ par jour selon l'état de l'herbe au pâturage et la disponibilité du fourrage conservé. Sa consommation journalière moyenne était de $0,42,0,23$ et $0,24 \mathrm{~kg}$ de MS par jour au cours des trois années successives pour le lot $\mathrm{H}$ (soit une moyenne de $0,30 \mathrm{~kg}$ sur 3 ans) et $0,15,0,30,0,13 \mathrm{~kg}$ MS pour le lot $\mathrm{P}$ ( soit $0,11 \mathrm{~kg}$ de moyenne sur 3 ans). La consommation moyenne d'aliments concentrés $(0,61 \mathrm{~kg}$ MS par jour) était identique pendant les 3 années dans les 2 lots. Le lot $\mathrm{P}$ recevait un traitement antiparasitaire $(0,22 \mathrm{~mL} / \mathrm{kg}$ PV d'oxfenzadole) en fin de printemps et début Décembre.

La productivité des parcelles a été estimée sur les surfaces pâturées par des coupes de $5 \mathrm{~m}$ sur $10 \mathrm{~cm}$ d'herbe par mini-tondeuse avec une hauteur de coupe de $5 \mathrm{~cm}$ juste avant que les chèvres accèdent aux parcelles, et sur les surfaces non pâturées à partir des stocks constitués lors des fauches. La production laitière et la composition du lait étaient contrôlées chaque semaine au cours de 2 traites successives, le poids vif et l'état corporel tous les mois.

Il est toujours difficile d'évaluer de façon précise les quantités de foin réel- lement ingérées puisque les chèvres ont toujours tendance à sélectionner leur ingéré et à faire des refus (Morand-Fehr 2003). Dans les conditions de cette expérience, les refus ont été estimés à $20 \%$ du distribué à partir d' 1 à 2 sondages par mois. Le lot $\mathrm{H}$ a reçu en moyenne $767 \mathrm{~kg}$ de MS de foin par an et le lot P, $408 \mathrm{~kg}$ de MS. Sur les 3 ans, le bilan annuel entre production et utilisation de foin a été déficitaire de $93 \mathrm{~kg}$ MS par chèvre dans le lot $\mathrm{H}$ et il est équilibré pour l'herbe pâturée dans le lot P. Ainsi, avec des surfaces fourragères équivalentes pour les 2 lots, l'autonomie fourragère a été globalement atteinte dans le lot $\mathrm{P}$ alors que dans le lot $\mathrm{H}$ les récoltes en foin étaient globalement insuffisantes par rapport aux besoins; ce qui a nécessité d'avoir recours d'une part, à une utilisation plus importante de déshydratés $(110,4$ vs $34,1 \mathrm{~kg} /$ chèvre/an) et d'autre part, à l'achat de foins. En moyenne sur les 3 ans, les quantités de foin ingéré par chèvre et par jour, respectivement pour le lot $\mathrm{H}$ et le lot $\mathrm{P}$, ont été évaluées de 1,78 vs $0,96 \mathrm{~kg}$. En moyenne, les chèvres du lot $P$ sont sorties au pâturage pendant 228 jours par an. 
Tableau 2. Effet du système d'alimentation, au pâturage ou en chèvrerie, sur la production et la composition du lait de chèvre (expérience 1).

\begin{tabular}{|c|c|c|c|c|c|c|c|c|}
\hline Année & \multicolumn{2}{|c|}{1} & \multicolumn{2}{|c|}{2} & \multicolumn{2}{|c|}{3} & \multicolumn{2}{|c|}{ Moyenne } \\
\hline Lot ${ }^{*}$ & $\mathrm{H}$ & $\mathbf{P}$ & $\mathrm{H}$ & $\mathbf{P}$ & $\mathrm{H}$ & $\mathbf{P}$ & $\mathrm{H}$ & $\mathbf{P}$ \\
\hline $\begin{array}{l}\text { Production de lait } \\
\text { (kg/an/chèvre) }\end{array}$ & $\begin{array}{c}798^{a} \\
(167)^{* *}\end{array}$ & $\begin{array}{l}745^{b} \\
(151)\end{array}$ & $\begin{array}{l}814^{a} \\
(186)\end{array}$ & $\begin{array}{l}856^{\mathrm{b}} \\
(187)\end{array}$ & $\begin{array}{c}932 \\
(184)\end{array}$ & $\begin{array}{l}928 \\
(183)\end{array}$ & 845 & 843 \\
\hline $\begin{array}{l}\text { Taux butyreux } \\
\text { (g/kg lait) }\end{array}$ & $\begin{array}{l}34,1^{\mathrm{a}} \\
(3,91)\end{array}$ & $\begin{array}{l}35,2^{\mathrm{b}} \\
(3,65)\end{array}$ & $\begin{array}{r}31,3 \\
(5,73) \\
\end{array}$ & $\begin{array}{l}32,1 \\
(3,63) \\
\end{array}$ & $\begin{array}{r}32,5 \\
(4,61) \\
\end{array}$ & $\begin{array}{c}33,0 \\
(4,27) \\
\end{array}$ & 32,6 & 33,4 \\
\hline $\begin{array}{c}\text { Taux protéique } \\
\text { (g/kg lait) }\end{array}$ & $\begin{array}{c}32,2 \\
(1,69)\end{array}$ & $\begin{array}{l}31,9 \\
(2,17) \\
\end{array}$ & $\begin{array}{l}30,2^{a} \\
(4,58)\end{array}$ & $\begin{array}{l}30,7^{\mathrm{b}} \\
(2,55)\end{array}$ & $\begin{array}{c}31,2 \\
(2,32) \\
\end{array}$ & $\begin{array}{l}31,1 \\
(2,16) \\
\end{array}$ & 31,2 & 31,6 \\
\hline
\end{tabular}

* Lot $\mathrm{H}$ maintenu en chèvrerie et recevant une ration à base de foin comme fourrage principal. Lot $\mathrm{P}$ conduit au pâturage et recevant une ration à base d'herbe comme fourrage principal ; ** L'écart-type est placé entre parenthèses.

Les valeurs affectées des lettres a et b en intra-année et inter-lot sont significativement différentes $(P<0,05)$.

Les valeurs sans lettre ne sont pas significativement différentes.

Comme l'indique le tableau 2, la production laitière moyenne des 2 lots $\mathrm{H}$ et $P$ sur trois ans est très voisine. En effet, la production de lait du lot $\mathrm{H}$ en première année et celle du lot $\mathrm{P}$ en deuxième année sont significativement plus élevées et se compensent alors qu'en troisième année, aucune différence n'est significative entre les productions annuelles de lait des 2 lots.

L'augmentation de la production laitière des 2 lots au cours des 3 années expérimentales s'explique par l'âge des animaux ; la première année, les chèvres sont essentiellement des primipares et la troisième, elles sont en $1^{\text {ere }}, 2^{\text {eme }}$ ou $3^{\text {ème }}$ lactation.

Les taux butyreux et protéique des lots $\mathrm{P}$ et $\mathrm{H}$ ne sont pas significativement différents en moyenne sur les trois années, même si le taux butyreux et le taux protéique du lot $\mathrm{P}$ sont significativement supérieurs respectivement en première et deuxième année.

Figure 1. Production laitière moyenne par chèvre lors de la deuxième année de la comparaison entre des chèvres alimentées en "chèvrerie» et au "pâturage» (expérience 1).

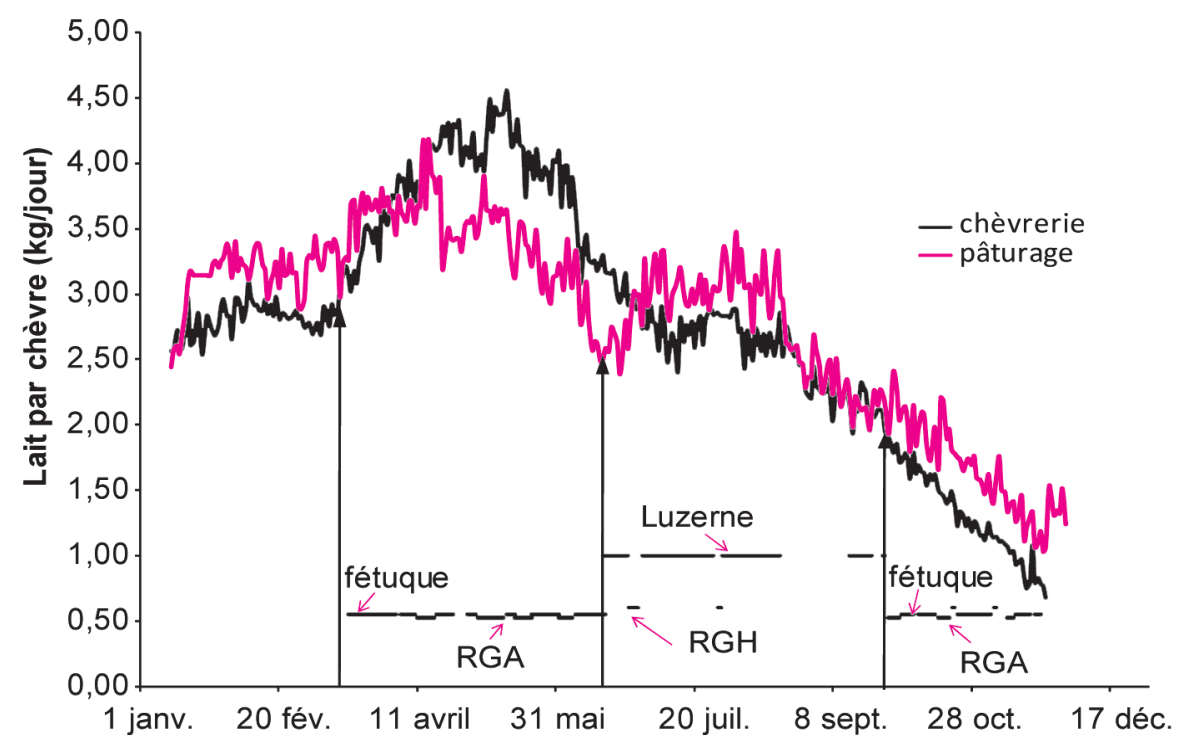

pâturage de graminées au printemps a eu pour conséquence au cours des 2 premières années, une baisse significative de production laitière (respectivement pendant les années 1 et 2 : - $0,39 \mathrm{~kg} /$ chèvre/jour $(\mathrm{P}<0,01)$ et - $0,42 \mathrm{~kg} /$ chèvre/jour $(\mathrm{P}<0,001))$, de poids vif et d'état corporel dans le lot $\mathrm{P}$ alors qu'en automne, l'effet a été inverse sur la production de lait $(+0,14$ et $+0,25 \mathrm{~kg} /$ chèvre/jour) comme le montre la figure 1 qui rapporte l'évolution de la production laitière des 2 lots au cours de la deuxième année. Par ailleurs, l'année 3 se distingue des années précédentes par un faible écart de production entre les 2 lots lors de l'utilisation de graminées au printemps $(3,74$ vs $3,53 \mathrm{~kg}$ de lait), ceci étant dû à une meilleure maîtrise de la pousse de l'herbe et à une pratique fréquente du pâturage simultané de différentes espèces (fétuque + raygrass anglais ou fétuque + ray-grass hybride + trèfle violet). Le passage d'un pâturage de graminées à celui de légumineuses se traduit par une remontée de la production laitière à la fin du printemps. En conséquence, la mobilisation des réserves corporelles est plus prononcée dans le lot $\mathrm{P}$ que dans le lot $\mathrm{H}$ au printemps, suivie d'une reconstitution de ces réserves plus rapide au cours du passage sur légumineuses. L'allongement de la durée d'exploitation des légumineuses au printemps, et de graminées en automne a permis de réduire les différences de production entre les 2 lots.

Par ailleurs, il faut remarquer que dans le contexte agro-climatique du sud de l'Ardèche, la récolte fourragère sous forme de foin ne permet pas d'exploiter correctement les repousses parfois abondantes en fin d'automne. Il s'en suit que la valorisation des surfaces est bien meilleure lorsque la fenaison n'intervient que pour la conservation des excédents de printemps comme cela a été le cas pour le lot $\mathrm{P}$. 
Ainsi, un système alimentaire à base de pâturage où l'herbe ingérée couvre nettement plus de la moitié des besoins énergétiques journaliers des chèvres (46 à 74\% selon l'année et le niveau de production laitière) permet des productions individuelles moyennes proches de $1000 \mathrm{~kg}$ de lait par an pour des animaux âgés de 1 à 3 ans comme en troisième année et une production voisine de $800 \mathrm{~kg}$ pour les primipares âgées d'un an comme en première année. Cette expérience a permis en outre de mettre en évidence trois points importants qui ont été respectés quotidiennement pour atteindre ce résultat: apport externe de foin de bonne qualité quand la disponibilité d'herbe sur la pâture est réduite, mise à l'herbe sur 3 semaines environ en réduisant progressivement l'apport de foin, exploitation rigoureuse des pâtures de graminées et de légumineuses en pâturage tournant à l'opposé d'une exploitation aléatoire comme c'est malheureusement encore parfois le cas en France. De plus, la valorisation du pâturage en arrière-saison permet de limiter les apports de foin à l'auge alors qu'il est difficile dans des périodes où les conditions climatiques sont incertaines, de réaliser des stocks de foin. Globalement, l'autonomie fourragère dans ces conditions, est plus facile à obtenir avec un système basé sur le prélèvement direct des fourrages cultivés par les chèvres, qu'avec un système basé sur l'utilisation exclusive de foin.

\section{2 / En pâturage continu (expérience 2)}

A la suite de l'expérience 1 , il a été décidé de confirmer ces résultats dans des conditions expérimentales différentes avec des chèvres multipares produisant $1100 \mathrm{~kg}$ de lait par an, pendant une durée courte (100 jours) et en pâturage continu. Ce pâturage se caractérise par de grandes parcelles de 1 à 2 ha, un chargement instantané de 20 à 30 chèvres par ha et une hauteur d'herbe maintenue à 6-8 $\mathrm{cm}$. Cette expérience (Van Quackebeke et al 1996b) a été conduite avec le même dispositif que l'expérience 1 et deux lots de 51 chèvres âgées de 2 ans ou plus et en pleine lactation (du $60^{\text {eme }}$ jour au $160^{\text {eme }}$ jour de lactation) : le lot $\mathrm{P}$ au pâturage et le lot $\mathrm{H}$ en chèvrerie.

Les productions de lait, les taux butyreux et protéique des 2 lots ont des valeurs proches, jamais significativement différentes malgré une légère tendance à ce que les productions du lot $\mathrm{P}$ soient supérieures (tableau 3). Cette expérience confirme les résultats de l'expérience 1 sur des chèvres multipares produisant environ $1080 \mathrm{~kg}$ de lait par an dans un troupeau dont l'effectif n'était plus en augmentation et utilisant un pâturage continu.

Tableau 3. Effet du système d'alimentation, au pâturage ou en chèvrerie, sur la production et la composition du lait de chèvre (expérience 2).

\begin{tabular}{|l|l|c|}
\hline \multicolumn{1}{|c|}{ Lot $^{*}$} & \multicolumn{1}{|c|}{ H } & P \\
\hline Production de lait (kg/jour/chèvre) & $3,99(1,04){ }^{* *}$ & $4,29(1,03)$ \\
\hline Taux butyreux (g/kg lait) & $30,7(3,90)$ & $32,8(3,46)$ \\
\hline Taux protéique (g/kg lait) & $30,6(2,12)$ & $31,5(2,13)$ \\
\hline
\end{tabular}

* Lot $\mathrm{H}$ maintenu en chèvrerie et recevant une ration à base de foin comme fourrage principal. Lot $\mathrm{P}$ conduit au pâturage et recevant une ration à base d'herbe comme fourrage principal. ** L'écart-type est placé entre parenthèses.

Dans des conditions climatiques tropicales, Soryal et al (2004) ont observé une diminution significative de production laitière de $15 \%$ et une très faible augmentation des taux quand les chèvres sont au pâturage. Fedele et al (1997) ont obtenu des résultats comparables dans des conditions méditerranéennes. Min et al (2005) ont enregistré des résultats divergents pendant deux années successives sur deux lots de chèvres recevant $0,66 \mathrm{~kg}$ de concentrés par litre de lait produit au dessus d'une production de $1,5 \mathrm{~kg}$. La première année, la production laitière du lot Chèvrerie était nettement supérieure à celle du lot Pâturage ; la seconde année, les productions des deux lots étaient très voisines. Les moins bons résultats ou les résultats contradictoires entre années obtenus par ces auteurs en utilisant des systèmes à base de pâturage semblent dus, à partir de l'analyse de Morand-Fehr (2005), au déficit alimentaire pendant certaines périodes de sécheresse, non compensé par des apports complémentaires de fourrages à l'auge comme c'était le cas au Pradel.

\section{4 / Comparaison du pâtura- ge continu et du pâturage tournant (expérience 3)}

L'expérience 3 (Van Quackebeke et al 1997) s'est déroulée sur 2 années successives $(1996,1997)$ afin de comparer 2 systèmes d'exploitation du pâturage par les chèvres : le pâturage tournant et le pâturage continu sur des prairies monospécifiques. Le niveau de fertilisation des parcelles pâturées était équivalent dans les 2 systèmes : 130, 90, 120 unités de N P K. L'expérience durait 91 et 112 jours au printemps de Mars (mise à l'herbe) à Juin respectivement la première et la seconde année.

Cent vingt chèvres âgées de 1 à 7 ans sont réparties en 2 lots de 60 chèvres, équilibrés au début de l'expérience sur la production laitière, le poids vif et l'état corporel. Le lot $\mathrm{T}$ est conduit au pâturage tournant sur 13 parcelles monospécifiques de 0,21 à 0,35 ha, à base de ray-grass hybride, de ray-grass anglais, de dactyle ou de fétuque élevée. Le chargement instantané est élevé et varie de 140 à 260 chèvres par ha, en alternant des courtes périodes de pâturage ( 1 à 4 jours par parcelle) et une période de repousse variable de 15 à 30 jours. Les chèvres entrent sur une parcelle quand la hauteur de l'herbe sur la parcelle est de 8 à $15 \mathrm{~cm}$ pour le premier cycle et de 10 à $13 \mathrm{~cm}$ pour le deuxième. Les chèvres sortent quand les hauteurs sont respectivement en $1^{\text {er }}$ et $2^{\text {ème }}$ cycle de 5 à $9 \mathrm{~cm}$ et de 5 à $6 \mathrm{~cm}$ sans excéder 4 jours de présence. En dehors de ces plages, l'herbe est fauchée pour constituer des stocks. Le lot $\mathrm{C}$ est conduit en pâturage continu sur 3 parcelles de graminées de 0,9 à 1,7 ha ayant la même composition botanique que les 13 parcelles du lot $\mathrm{T}$. Le chargement instantané du lot $\mathrm{C}$ est faible : de 35 à 60 chèvres par ha. Les chèvres disposent de trois parcelles. Le choix de la parcelle du jour dépend de la hauteur de l'herbe en privilégiant la rotation des parcelles. Ces parcelles n'ont pas de temps de repos. Le gazon reste court car la faible quantité d'herbe disponible au mètre carré est compensée par la mise à disposition des chèvres d'une surface nettement plus importante. La surface globale attribuée à chaque lot au printemps est de 4,3 ha. L'apport de concentré est maintenu à $0,8 \mathrm{~kg}$ par chèvre et par jour dans les 2 lots pendant toute l'expérience.

La production laitière de chaque lot est contrôlée chaque jour. La production laitière, le taux butyreux et le taux protéique de chaque chèvre sont mesurés une fois par semaine au cours de 2 traites successives. La hauteur de l'herbe est contrôlée à l'entrée et à la sortie des parcelles en pâturage tournant, et chaque semaine en pâturage continu.

Aucune différence significative n'a pu être mise en évidence sur la production et la composition du lait des lots $\mathrm{T}$ et $C$ (tableau 4). Des résultats identiques ont déjà été enregistrés avec des vaches laitières (Munger et Jans 2002, Pulido et Leaver 2003). 
Tableau 4. Effet du mode de conduite du pâturage, tournant ou continu, sur la production et la composition du lait de chèvre (expérience 3).

\begin{tabular}{|l|c|c|c|c|c|c|}
\hline \multicolumn{1}{|c|}{ Année } & \multicolumn{2}{c|}{$\mathbf{1}$} & \multicolumn{2}{c|}{$\mathbf{2}$} & \multicolumn{2}{c|}{ Moyenne } \\
\hline \multicolumn{1}{|c|}{ Lot * $^{*}$} & $\mathbf{T}$ & $\mathbf{C}$ & $\mathbf{T}$ & $\mathbf{C}$ & $\mathbf{T}$ & $\mathbf{C}$ \\
\hline Production de lait (kg/jour/chèvre) & $3,87(0,94) * *$ & $3,83(1,07)$ & $4,23(1,16)$ & $4,19(1,13)$ & 4,06 & 4,01 \\
\hline Taux butyreux (g/kg lait) & $34,2(7,92)$ & $34,6(8,29)$ & $34,0(3,79)$ & $34,4(4,99)$ & 34,1 & 34,5 \\
\hline Taux protéique (g/kg lait) & $31,8(6,54)$ & $31,8(7,02)$ & $31,7(2,30)$ & $31,9(8,75)$ & 31,8 & 31,9 \\
\hline
\end{tabular}

Lot $T$ : chèvres conduites en pâturage tournant.

Lot $C$ : chèvres conduites en pâturage continu.

** L'écart-type est placé entre parenthèses.

$\mathrm{Au}$ cours de cette expérience, deux observations qui n'ont pu être quantifiées ont été mises en évidence :

- Les différents types de ray-grass utilisés (anglais et hybride) semblent toujours favorables à une bonne production laitière alors que le dactyle et la fétuque ont tendance à la diminuer. Deux raisons principales peuvent expliquer ces constatations, d'une part, une palatabilité plus élevée des ray-grass qui se traduit par une amélioration de l'ingestion (de Simiane et al 1981, Masson et Hacala 1985). Néanmoins, lorsque l'herbe est rare et le taux de matière sèche élevé, l'effet de l'espèce végétale tend à s'estomper; ce fut le cas lors des 2 premiers cycles de la deuxième année. D'autre part, le ray-grass a une meilleure digestibilité par rapport au dactyle et à la fétuque (INRA 2007).

- Une herbe trop haute supérieure à $15 \mathrm{~cm}$ qui s'observe plus fréquemment en pâturage tournant entraîne un tri plus important des chèvres et une qualité des repousses médiocre. Une hauteur d'herbe inférieure à $7 \mathrm{~cm}$, fréquente en pâturage continu, a tendance à limiter l'ingestion mais les chèvres réagissent en augmentant leur temps de pâturage. Une hauteur entre 7 et $14 \mathrm{~cm}$ semble favorable à un niveau d'ingestion optimal quand les repousses sont feuillues dans le cas d'un pâturage tournant et entre 5 et $8 \mathrm{~cm}$ en pâturage continu.

En conclusion de cette expérience, les performances très voisines des chèvres conduites en pâturage tournant et en pâturage continu peuvent s'expliquer par le suivi minutieux et journalier de l'état des parcelles et par la gestion précise du pâturage qui en a découlé. Cette gestion rigoureuse des parcelles est plus facile à mettre en œuvre dans le cas du pâturage continu car l'observation d'une seule parcelle est plus facile et le plus souvent, la qualité des repousses en pâturage tournant est plus difficile à prévoir. Mais en moyenne sur les deux années, le pâturage tournant a permis de collecter plus d'excédents sous forme de foin ( $20 \%$ en plus). En outre, d'après nos calculs dans les conditions du Pradel, le coût en main-d'œuvre est sensiblement inférieur en pâturage continu en raison d'une durée d'observation nécessaire du couvert végétal plus courte.

\section{5 / Effet de la date de la mise à l'herbe (expérience 4)}

Comme le choix de la date de la mise à l'herbe est une décision essentielle dans la conduite des chèvres au pâturage, l'expérience 4 qui a déjà été décrite par Lefrileux et al (2000) compare une date de mise à l'herbe de référence aux environs du 31 Mars qui est fréquemment pratiquée par les éleveurs de chèvres dans la région, et une mise à l'herbe précoce, un mois auparavant quand la hauteur de l'herbe est faible, environ $8 \mathrm{~cm}$. Le but principal de cette expérience était d'estimer les effets du passage d'un régime hivernal à base de foin en chèvrerie à un régime de printemps à base d'herbe verte sur la production et la composition du lait de chèvre, selon que la mise à l'herbe est tardive ou précoce.

L'expérience a duré 120 jours à partir du début de la mise à l'herbe précoce. Deux lots de 60 chèvres équilibrés sur les mêmes paramètres que pour les expériences précédentes ont été constitués : le lot T est mis au pâturage quand l'herbe atteint en moyenne $15 \mathrm{~cm}$ de haut, à la date du 31 Mars, le lot $\mathrm{P}$ quand l'herbe atteint seulement $7 \mathrm{~cm}$ environ, un mois avant le lot $\mathrm{T}$. La transition du régime hivernal au pâturage dure 1 mois pour le lot $\mathrm{P}$ et seulement 10 jours pour le lot T. Avant la mise à l'herbe, toutes les chèvres reçoivent du foin et $800 \mathrm{~g}$ d'aliments concentrés par jour. Pendant la mise à l'herbe, les chèvres des 2 lots reçoivent $800 \mathrm{~g} / \mathrm{j}$ d'un aliment concentré commercial (1,0 UFL et $260 \mathrm{~g}$ de MAT/kg de MS) et la quantité de foin offerte chaque jour diminue progressivement. Les contrôles et les mesures ont été réalisés dans les mêmes conditions sur les chèvres et le lait que dans l'expérience 3.

Les résultats sur les performances moyennes des chèvres pendant toute l'expérience mettent en évidence un effet significativement positif d'une mise à l'herbe précoce sur la production de lait et le taux butyreux alors que l'effet sur le taux protéique n'est pas significatif (tableau 5). Cet effet est largement dû à la période où le lot $\mathrm{P}$ était au

Tableau 5. Effet d'une mise à l'herbe précoce ou tardive sur la production et la composition du lait de chèvre au pâturage (expérience 4).

\begin{tabular}{|l|l|c|}
\hline \multicolumn{1}{|c|}{ Lot $^{*}$} & \multicolumn{1}{|c|}{$\mathbf{T}$} & $\mathbf{P}$ \\
\hline Production de lait $^{* *}$ (kg/jour/chèvre) & $3,80^{\mathrm{a}}(0,99)^{* * *}$ & $3,98^{\mathrm{b}}(0,96)$ \\
\hline Taux butyreux $^{* *}$ (g/kg lait) & $35,4^{\mathrm{a}}(4,14)$ & $36,7^{\mathrm{b}}(4,57)$ \\
\hline Taux protéique $^{* *}$ (g/kg lait) & $31,4(2,34)$ & $31,6(2,47)$ \\
\hline
\end{tabular}

* Lot $\mathrm{T}$ avec une mise à l'herbe tardive (31 Mars), Lot $\mathrm{P}$ avec une mise à l'herbe précoce ( $1^{\mathrm{er}}$ Mars).

** Valeurs moyennes des performances laitières pendant 120 jours à partir du premier jour de la mise à l'herbe précoce du lot $P ;{ }^{* * *} L$ 'écart-type est placé entre parenthèses.

Entre les lots, les valeurs avec les lettres a et b sont significativement différentes $(P<0,05)$. 
pâturage avec une ration mixte à base d'herbe pâturée et de fourrage sec et le lot $\mathrm{T}$ uniquement avec du foin. Pendant cette période, les quantités moyennes de matière sèche ingérée en chèvrerie étaient respectivement pour les lots $\mathrm{P}$ et $\mathrm{T}$, de 2,2 vs 3,3 kg de foin par chèvre et par jour et les productions laitières de 4,58 vs $4,08 \mathrm{~kg}(\mathrm{P}<0,001)$. Des résultats comparables ont été observés sur vaches laitières soumises à une mise à l'herbe précoce (Sayers et Mayne 2001).

La mise à l'herbe précoce par rapport à la mise à l'herbe tardive a permis, à surface égale, d'économiser environ $36 \mathrm{~kg}$ de MS de foin distribué à l'auge par chèvre, sachant que les surfaces disponibles et les quantités de concentrés étaient identiques. Cette économie de distribution est à moduler sachant que dans cet essai, les quantités de fourrages récoltés étaient plus importantes quand la date de mise au pâturage était plus tardive (environ $+30 \%$ ).

Cette expérience met en évidence l'intérêt de procéder à une mise à l'herbe précoce. Cet intérêt doit être modulé en fonction de la valeur alimentaire de la ration hivernale. La mise à l'herbe précoce aura un effet d'autant plus favorable sur les performances laitières que la qualité du foin de la ration hivernale sera médiocre comme cela a été déjà observé chez les vaches (Peyraud et al 1995).

Il faut aussi noter que dans le cas d'une mise à l'herbe tardive sur des prairies où la hauteur d'herbe est déjà de 10 à $15 \mathrm{~cm}$, les chèvres préfèrent l'herbe verte au foin, ce qui pousse à une transition rapide entre les 2 régimes. A l'inverse, dans le cas d'une mise à l'herbe précoce, la faible hauteur de l'herbe $(6$ à $8 \mathrm{~cm})$ liée à une disponibilité réduite limite l'ingestion de cette herbe, ce qui incite plutôt à procéder à une transition plus longue.

Des résultats comparables ont été enregistrés en ovins où la mise à l'herbe précoce et même le pâturage hivernal sont pratiqués quand les conditions agroclimatiques s'y prêtent (Gautier et Moulin 2004).

\section{6 / Effets du niveau d'ap- port d'aliments concentrés au pâturage \\ (expériences 5, 6, 7,8)}

Pour valoriser au maximum la part d'herbe pâturée dans le régime alimentaire des chèvres laitières hautes productrices, tout en obtenant les performances laitières maximales, il est essentiel pour l'éleveur de définir le niveau des apports d'aliments concentrés en fonction de la qualité du couvert végétal.

Quatre expériences successives ont été réalisées sur ce sujet. La première (expérience 5) a duré 12 semaines (début Avril à fin Juin) et a été conduite en pâturage continu dans les mêmes conditions que le lot $\mathrm{C}$ de l'expérience 3. 120 chèvres utilisaient la prairie en pâturage continu avec une hauteur d'herbe comprise entre 5 et $11 \mathrm{~cm}$. L'objectif de cette expérience était de comparer 5 niveaux d'aliments concentrés : 0,28, 0,55, 0,80, 1,05, 1,30 kg/jour en utilisant 5 lots de 20 chèvres et en maintenant 1'apport de PDI issues des concentrés constant à $155 \mathrm{~g} / \mathrm{j}$. En réalité, cela a été obtenu en faisant varier dans les rations la proportion de 2 aliments concentrés se distinguant par leur richesse en protéines, le premier $(1,01$ $\mathrm{UFL} / \mathrm{kg}$ de MS, $330 \mathrm{~g}$ de PDIN/kg de MS, $349 \mathrm{~g}$ de PDIE/kg de MS) et le deuxième $(1,06 \mathrm{UFL} / \mathrm{kg}$ de MS, $132 \mathrm{~g}$ de PDIN/kg de MS, $136 \mathrm{~g}$ de PDIE/kg de MS), à l'exception du premier niveau $(0,28 \mathrm{~kg})$ pour lequel les chèvres n'ont reçu que $90 \mathrm{~g}$ de PDI par jour par l'intermédiaire du concentré.

La deuxième expérience (expérience 6) a été conduite comme l'expérience 5 de début Avril à fin Juin en pâturage continu avec deux niveaux d'apport de concentrés. Cent vingt chèvres ont été réparties en 2 lots recevant respectivement 0,65 et $1,3 \mathrm{~kg}$ d'un aliment du commerce (1 UFL/kg et $182 \mathrm{~g}$ de $\mathrm{MAT} / \mathrm{kg}$ d'aliment correspondant à $145 \mathrm{~g}$ de PDIN/kg de MS et $137 \mathrm{~g}$ de $\mathrm{PDIE} / \mathrm{kg}$ de MS).

La troisième expérience (expérience 7) a été réalisée au printemps entre le $60^{\text {eme }}$ et le $160^{\text {eme }}$ jour de lactation dans les mêmes conditions expérimentales que le lot $\mathrm{P}$ de l'expérience 2 et sur 34 chèvres, réparties en deux lots de 17 chèvres équilibrés au début de l'expérience. Les lots $\mathrm{B}$ et $\mathrm{H}$ recevaient 0,5 et $0,8 \mathrm{~kg}$ par jour respectivement d'un aliment concentré du commerce $(1,17 \mathrm{UFL} / \mathrm{kg}$ de MS, $264 \mathrm{~g}$ de MAT/kg de MS, et à $183 \mathrm{~g}$ de PDIN/kg de MS et $172 \mathrm{~g}$ de PDIE/kg de MS).

La quatrième expérience (expérience 8) déjà décrite par Lefrileux et al (2005) a été réalisée pendant 125 jours au printemps avec 4 lots de 27 chèvres dans les mêmes conditions expérimentales que l'expérience 7. Les lots HM et HC recevaient respectivement $1 \mathrm{~kg}$ de maïs (1,20 UFL/kg de MS, $74 \mathrm{~g}$ de PDIN/kg de MS et $97 \mathrm{~g}$ de PDIE $/ \mathrm{kg}$ de MS) et d'aliment concentré du commerce $(1,01$ UFL/kg de MS, $190 \mathrm{~g}$ de PDIN/kg de $\mathrm{MS}, 158 \mathrm{~g}$ de PDIE $/ \mathrm{kg}$ de MS), et les lots $\mathrm{BM}$ et $\mathrm{BC}, 0.5 \mathrm{~kg}$ seulement des mêmes aliments.

$\mathrm{Au}$ cours de ces quatre expériences, les analyses effectuées sur divers échantillons des principales espèces fourragères exploitées (ray-grass anglais, raygrass hybride et dactyle) ont montré que les valeurs énergétiques et azotées restaient élevées $(1,1$ à $0,95 \mathrm{UFL} / \mathrm{kg}$ de $\mathrm{MS}, 120$ à $130 \mathrm{~g}$ de PDIE/kg de MS, 120 à $140 \mathrm{~g}$ de PDIN/kg de MS) de début Avril à fin Mai, mais que fin Mai ou début Juin, elles chutaient à environ $0,80 \mathrm{UFL} / \mathrm{kg}$ de $\mathrm{MS}, 100 \mathrm{~g}$ de PDIE $/ \mathrm{kg}$ de MS et $90 \mathrm{~g}$ de PDIN $/ \mathrm{kg}$ de MS. En Avril, l'herbe contenait 60 à $80 \%$ de limbes alors qu'en Juin, cette proportion était nettement plus faible, aux alentours de $40 \%$.

Tableau 6. Effet de l'apport d'aliments concentrés sur la production laitière des chèvres au pâturage (expériences 5, 6, 7, 8).

\begin{tabular}{|c|c|c|c|}
\hline \multirow{2}{*}{ Expérience } & \multicolumn{2}{|c|}{ Concentré } & $\begin{array}{c}\text { Augmentation de la production } \\
\text { de lait par 100 g de concentré } \\
\text { (g lait) }\end{array}$ \\
\hline & \multicolumn{2}{|c|}{$\begin{array}{c}\text { Minimum } \\
\mathrm{kg} / \mathrm{j} / \text { chèvre }\end{array}$} & $\begin{array}{c}\text { Maximum } \\
\mathrm{kg} / \mathrm{j} / \mathrm{chèv}\end{array}$ \\
\hline 5 & 0,26 & 1,3 & $46^{*}$ \\
\hline 6 & 0,65 & 1,3 & $87^{*}$ \\
\hline 7 & 0,50 & 0,8 & $63^{*}$ \\
\hline 8 (lot maïs) & 0,50 & 1,0 & 20 \\
\hline 8 (lot concentré du commerce) & 0,50 & 1,0 & $78^{*}$ \\
\hline
\end{tabular}

${ }^{*}$ Augmentation significative de la production de lait par $100 \mathrm{~g}$ de concentré $(P<0,05)$. 
Figure 2. Effet de la quantité d'aliment concentré sur la production laitière des chèvres au pâturage.

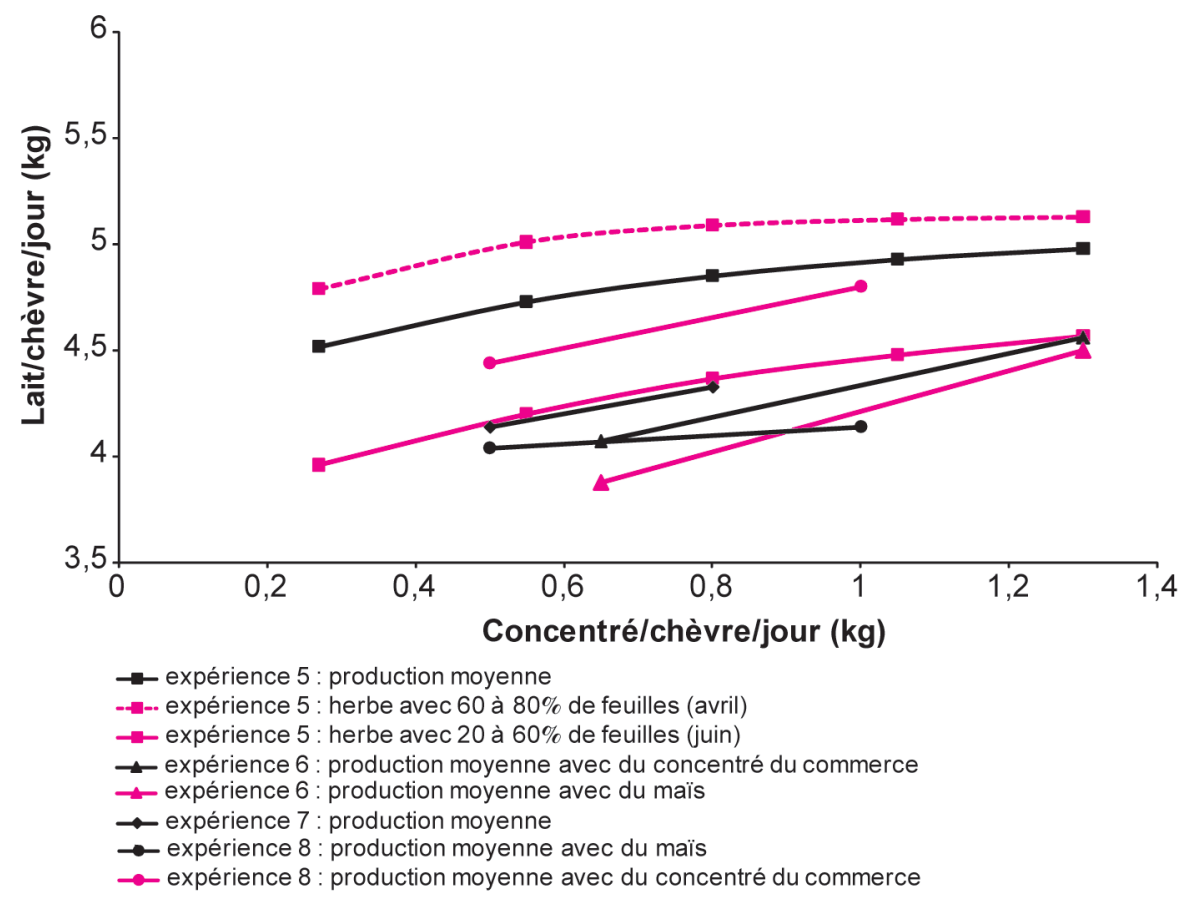

Les figures 2, 3a, $3 \mathrm{~b}$ et le tableau 6 rapportent les productions et la composition du lait au cours de ces expériences. L'augmentation de l'apport d'aliments concentrés améliore la production laitière de façon significative sauf dans pour les lots BM et HM recevant du maïs dans l'expérience 8 pour lesquels l'effet du niveau de concentré n'est pas significatif (tableau 6).

L'effet de l'augmentation de l'apport d'aliment concentré sur la production laitière est plus important lorsque le niveau d'apport d'aliment concentré est faible $(0,3 \mathrm{~kg} /$ jour $)$ par rapport à un niveau élevé (1,3 kg/jour) (figure 2). Ainsi dans l'expérience 5, l'apport de $100 \mathrm{~g}$ de concentré entre 0,26 et $0,52 \mathrm{~kg}$ élève la production de lait de $81 \mathrm{~g}$, et entre 1,04 et $1,3 \mathrm{~kg}$, de $19 \mathrm{~g}$ seulement. Des résultats très voisins concernant l'effet du niveau de concentré sur l'ingestion d'herbe et la production laitière ont aussi été enregistrés chez les vaches laitières (Peyraud et al 1998). Cette observation est probablement due en grande partie au fait que l'apport croissant de concentré réduit l'ingestion d'herbe. Cela est confirmé par la réduction d'une à deux heures du temps de pâturage des chèvres consommant $1,3 \mathrm{~kg}$ au lieu de $0,26 \mathrm{~kg} / \mathrm{j}$ de concentré dans l'expérience 5 .

De plus, comme le montre la figure 2, l'amélioration de la production laitière dans l'expérience 5 avec la même augmentation d'aliment concentré, est relativement plus élevée avec de l'herbe à un stade végétatif avancé de moyenne qualité (c'est le cas en Juin) qu'à un stade plus jeune de bonne qualité (en Avril); ce qui confirme les résultats de Min et al (2005). Ainsi, la réponse des chèvres doit dépendre de leur bilan énergétique comme l'ont montré Coulon et Rémond (1991) chez les vaches.

Des résultats comparables ont été rapportés avec des chèvres par Soryal et al (2004) dans des conditions tropicales. Ils ont observé pour des chèvres moins productives $(2,8 \mathrm{~kg}$ de lait par jour en moyenne), une augmentation significative de $0,8 \mathrm{~kg}$ de lait par jour quand la distribution journalière d'aliments concentrés passe de 0 à $0,9 \mathrm{~kg}$ avec peu d'effets sur la composition du lait.

Les taux butyreux et protéique ne varient pas significativement avec l'apport de concentré dans les 4 expériences (figure $3 a$ et b). Seuls, les effets de l'apport d'aliment concentré sur le taux butyreux dans l'expérience 8 sont significatifs $(\mathrm{P}<0,05)$. Toutefois, la figure $3 \mathrm{a}$ et $\mathrm{b}$ montrent plutôt une tendance à une augmentation des taux quand la consommation d'aliments concentrés croît, en particulier de 0,5 à $1 \mathrm{~kg}$ de concentré par jour.

A partir des observations faites au cours de ces quatre expériences, il semble que la qualité de l'herbe au pâturage pourrait avoir une plus grande influence sur les performances de la chèvre haute productrice de lait que la quantité d'aliments concentrés distribuée. Une ration composée d'herbe de bonne qualité et d'aliment concentré en quantité limitée serait plus efficace pour améliorer la production laitière qu'une ration d'herbe de médiocre qualité avec une quantité d'aliment concentré supérieure à $0,8 \mathrm{~kg}$ par jour. En effet, il semble peu efficace d'apporter plus de $0,8 \mathrm{~kg}$ d'aliment concentré par jour au pâturage sur les plans technique et surtout économique. Mais en dessous de $0,8 \mathrm{~kg}$, un apport d'aliment concentré tend à plus améliorer la production laitière lorsque l'herbe est de médiocre qualité que lorsqu'elle est de bonne qualité. Fedele et al (1993) ont observé avec des chèvres à potentiel laitier plus faible dans le Sud de l'Italie qu'un haut niveau de complémentation abaissait l'ingestion de l'herbe pâturée et n’améliorait pas la production de lait. En pâturage rationné dans les conditions d'élevage du Centre-Est de la France, Masson et Hacala (1985) obtenaient même une production de lait plus faible.

\section{7 / Effets des caractéristi- ques énergétiques et azotées des aliments concentrés} (expériences 4, 6, 8,9)

Dans le cas d'une conduite au pâturage des chèvres laitières, non seulement le niveau d'apports d'aliments concentrés doit être établi selon le potentiel de production des chèvres, la qualité et le stade végétatif de l'herbe, mais la nature des sources énergétiques (glucides rapidement ou lentement dégradables dans le rumen), la teneur en matières azotées et la nature des sources azotées (faiblement ou fortement dégradables dans le rumen) dans l'apport d'aliments concentrés distribués quotidiennement doivent aussi être mieux définies.

\section{1 / Nature de la source éner- gétique de l'aliment concentré (expérience 6)}

Les 2 lots de 60 chèvres de l'expérience 6 ont été divisés chacun en deux souslots de 30 chèvres. Les sous-lots 1,30 A et $0,65 \mathrm{~A}$ reçoivent respectivement $1,30 \mathrm{~kg}$ et $0,65 \mathrm{~kg}$ par jour d'un aliment riche en amidon (527 g/kg de MS), pauvre en glucides pariétaux (130 g de $\mathrm{NDF} / \mathrm{kg}$ de $\mathrm{MS}$ ) et en matières grasses (22 $\mathrm{g}$ d'EE/ $\mathrm{kg}$ de MS), et les sous-lots $1,30 \mathrm{~F}$ et $0,65 \mathrm{~F}$, les mêmes quantités mais d'un aliment au contraire pauvre en amidon (258 g/kg de MS), riche en glucides pariétaux (308 g de NDF/kg de $\mathrm{MS})$ et en matières grasses ( $99 \mathrm{~g}$ d'EE $/ \mathrm{kg}$ de MS), les aliments A et $\mathrm{F}$ étant isoénergétiques (1,0 UFL $/ \mathrm{kg}$ de MS) et isoprotéiques (145 $\mathrm{g}$ de $\mathrm{PDIN} / \mathrm{kg}$ de MS et $137 \mathrm{~g}$ de PDIE/kg de MS). Comme il n'y a pas d'interaction entre les effets du niveau de concentrés et de la nature de la source énergétique, chaque facteur peut être analysé séparément comme dans le tableau 7. 
Figure 3. Effet de la quantité d'aliment concentré sur la composition du lait des chèvres au pâturage : a) Taux protéique, b) Taux butyreux.
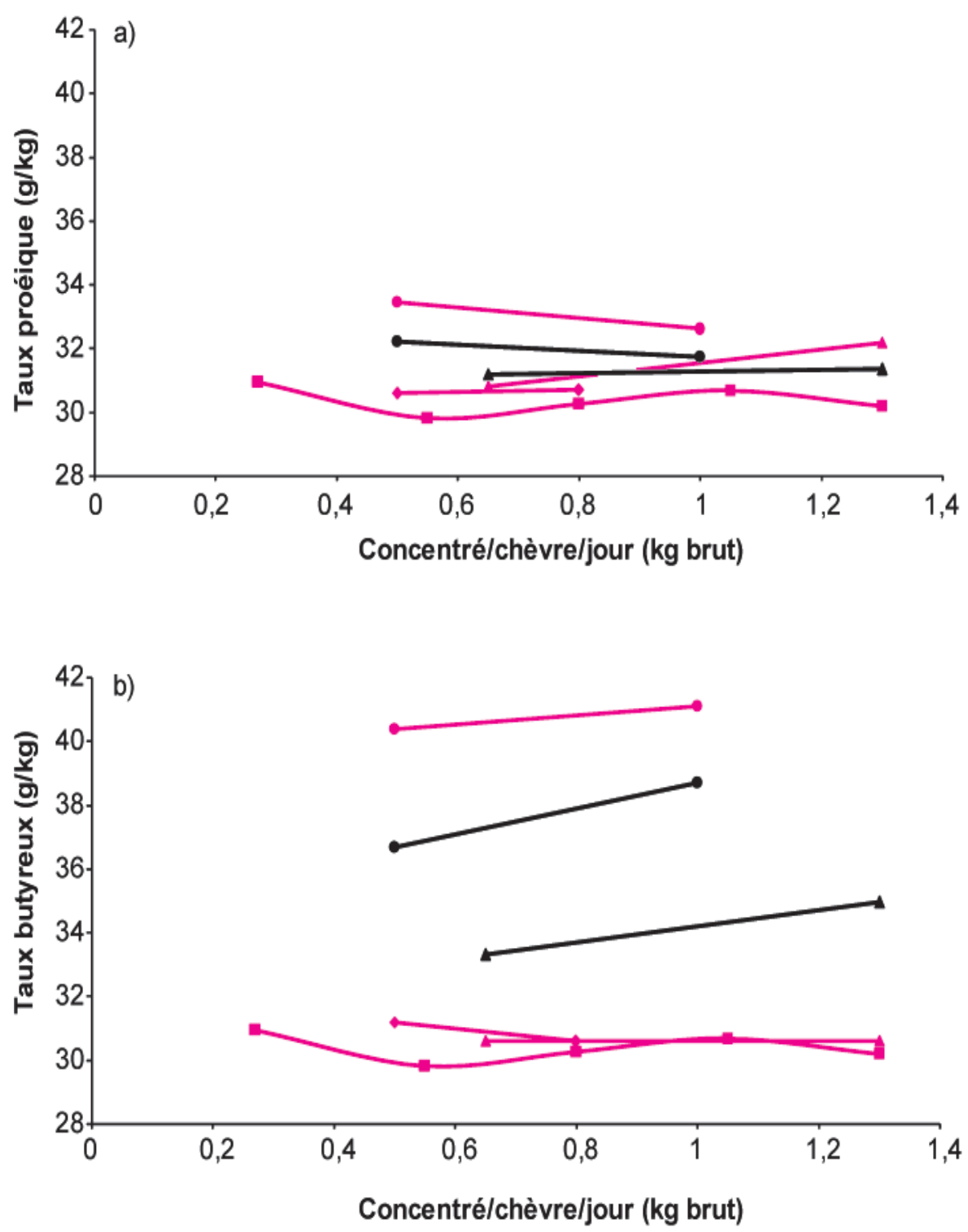

--expérience 5 production moyenne
^-expérience 6 concentré du commerce
„-expérience 7 concentré du commerce

¿expérience 6 maïs

-expérience 8 maïs

- $\rightarrow$ expérience 8 concentré du commerce
La production laitière et le taux protéique du lait (tableau 7) ne sont pas significativement influencés par la substitution iso-énergétique et iso-protéique de $50 \%$ de l'amidon de l'aliment concentré par le mélange fibres-lipides. Des résultats identiques ont été obtenus sur vaches laitières au pâturage recevant des concentrés riches en amidon ou en fibres (Delaby et Peyraud 1994, Wilson et al 2002). En revanche, le taux butyreux est significativement amélioré avec le concentré riche en fibres et en matière grasse. Une amélioration identique a été obtenue dans l'expérience 8 (figure $3 \mathrm{~b}$ ) quand l'aliment concentré contient $9 \%$ de lipides protégés. Ceuxci ne peuvent pas modifier les fermentations de rumen et notamment la cellulolyse puisqu'ils sont protégés. Cet effet sur le taux butyreux et en conséquence sur la quantité secrétée de matière grasse est donc dû à un apport supérieur de fibres et de matière grasse qui sont les deux sources alimentaires principales des précurseurs des acides gras du lait de ruminant, et en particulier de chèvre (Morand-Fehr et al 1991, Schmidely et al 2005).

\section{2 / Niveau azoté de l'aliment concentré (expériences 8 et 9)}

L'expérience 9 a été réalisée suivant le même protocole que l'expérience 7 avec 34 chèvres réparties en deux lots de 17 chèvres. Le lot $\mathrm{O}$ reçoit $0,8 \mathrm{~kg}$ d'orge et le lot $\mathrm{C} 0,8 \mathrm{~kg}$ d'un aliment concentré iso-énergétique du commerce $(1,11 \mathrm{UFL} / \mathrm{kg}$ de MS) mais plus riche en matières azotées totales $(253 \mathrm{~g}$ de $\mathrm{MAT} / \mathrm{kg}$ de MS) et en PDI (176 $\mathrm{g}$ de PDIN et $165 \mathrm{~g}$ de PDIE/kg de MS) que l'orge (110 g de MAT, $76 \mathrm{~g}$ de PDIN et $98 \mathrm{~g}$ de PDIE/kg de MS).

Selon le tableau 8, la substitution d'un aliment concentré du commerce plus riche en matière azotée à de l'orge améliore significativement la production

Tableau 7. Effet du niveau d'apport et de la nature de la source énergétique des aliments concentrés sur la production et la composition du lait des chèvres au pâturage (expérience 6).

\begin{tabular}{|c|c|c|c|c|c|}
\hline Apport en concentré $(\mathrm{kg} / \mathrm{j})$ & 0,65 & 0,65 & 1,3 & 1,3 & \multirow{2}{*}{$\begin{array}{c}\text { Signification } \\
\text { de l'effet de } \\
\text { la source } \\
\text { énergétique }\end{array}$} \\
\hline Nature de la source énergétique & Amidon & $\begin{array}{l}\text { Fibre + } \\
\text { Graisse }\end{array}$ & Amidon & $\begin{array}{l}\text { Fibre + } \\
\text { Graisse }\end{array}$ & \\
\hline Production de lait (kg/j/chèvre) & $\begin{array}{c}4,07 \\
(0,74) \text { * }\end{array}$ & $\begin{array}{c}3,88 \\
(0,64)\end{array}$ & $\begin{array}{c}4,56 \\
(0,76)\end{array}$ & $\begin{array}{c}4,50 \\
(0,94)\end{array}$ & NS \\
\hline Taux butyreux (g/kg lait) & $\begin{array}{c}30,6 \\
(3,28)\end{array}$ & $\begin{array}{c}33,3 \\
(3,84)\end{array}$ & $\begin{array}{c}30,6 \\
(4,35)\end{array}$ & $\begin{array}{c}35,0 \\
(5,19)\end{array}$ & ** \\
\hline Taux protéique (g/kg lait) & $\begin{array}{c}30,8 \\
(2,41)\end{array}$ & $\begin{array}{c}31,2 \\
(2,52)\end{array}$ & $\begin{array}{c}32,2 \\
(2,58)\end{array}$ & $\begin{array}{c}31,4 \\
(2,91)\end{array}$ & NS \\
\hline
\end{tabular}

NS $=$ Non Significatif.

* L'écart-type est placé entre parenthèses ; ** Significatif au seuil $P<0,01$. 
Tableau 8. Effet de la teneur azotée de l’aliment concentré sur les performances laitières des chèvres au pâturage (expérience 9).

\begin{tabular}{|l|c|c|}
\hline \multicolumn{1}{|c|}{ Lot $^{*}$} & $\mathbf{0}$ & $\mathbf{C}$ \\
\hline Teneur en PDIN de l'aliment concentré (g/kg MS) & 76 & 176 \\
\hline Production de lait (kg/j/chèvre) & $4,22^{\mathrm{a}}(1,18)^{* *}$ & $4,64^{\mathrm{b}}(1,02)$ \\
\hline Taux butyreux (g/kg lait) & $32,6(3,25)$ & $32,4(3,01)$ \\
\hline Taux protéique (g/kg lait) & $32,1(2,08)$ & $31,2(1,70)$ \\
\hline
\end{tabular}

* Lot $O$ : chèvres recevant $0,8 \mathrm{~kg}$ d'orge par jour, Lot $C:$ chèvres recevant $0,8 \mathrm{~kg}$ d'aliment concentré du commerce par jour.

** L'écart-type est placé entre parenthèses.

Entre les lots, les valeurs affectées des lettres a et $b$ sont significativement différentes $(P<0,05)$.

laitière de $10 \%$ alors que les taux butyreux et protéique gardent des valeurs très voisines. Ces résultats ont été observés au cours de toute l'expérience tant sur les chèvres fortes productrices (en moyenne 5,0 kg de lait par jour) que sur les faibles productrices $(3,3 \mathrm{~kg}$ de lait par jour). Ils pourraient s'expliquer par une amélioration de la digestibilité de la ration liée à l'apport des concentrés azotés comparativement aux concentrés énergétiques qui n'ont pas les mêmes effets sur la digestibilité.

Dans l'expérience 8 (Lefrileux et al 2005) dont le protocole expérimental a été décrit précédemment, les lots HM et $\mathrm{BM}$ recevaient 1 et $0,5 \mathrm{~kg}$ de maïs contenant $10 \%$ de MAT et les lots HC et $\mathrm{BC}$, la même quantité d'un aliment concentré du commerce contenant $26 \%$ de MAT et un apport supplémentaire de matière grasse. L'apport d'aliment concentré à la place du maïs améliore la production laitière de $10 \%$ à un niveau d'apport de $0,5 \mathrm{~kg}$ de concentré/jour et d'un peu moins de $20 \%$ avec $1 \mathrm{~kg}$ de concentré (figure 2). En revanche, le taux protéique n'est pas significativement influencé par l'apport supplémentaire de matière azotée dans le concentré. Le taux butyreux est significativement amélioré, très probablement influencé par l'ap- port supplémentaire de matière grasse présente dans l'aliment concentré. Ainsi, les effets d'une augmentation de la teneur en matière azotée de l'aliment concentré ont été comparables dans les expériences 8 et 9 .

Il est intéressant de noter dans les expériences 8 et 9 que l'apport azoté total des chèvres recevant l'aliment concentré du commerce excède d'environ $40 \%$, leurs besoins azotés totaux établis par l'INRA (Morand-Fehr et Sauvant 1989). Toutefois, l'application de la notion de besoin à un animal cible dans un lot, peut être délicate car elle dépend de l'hétérogénéité de ce lot. Dans ces deux expériences, les besoins azotés de $90 \%$ des chèvres étaient couverts.

\section{3 / Caractéristiques des sources azotées du concentré \\ (expérience 4)}

$\mathrm{Au}$ cours de l'expérience 4 testant l'effet d'une mise à l'herbe précoce ou tardive (cf. partie 5), chacun des 2 lots de 60 chèvres a été divisé en 2 sous-lots équilibrés qui recevaient chacun $0,8 \mathrm{~kg}$ d'un aliment concentré du commerce. Les deux aliments étaient iso-énergétiques $(1,1 \mathrm{UFL} / \mathrm{kg}$ de MS) et iso-azotés
(258 g de MAT/kg de MS) mais l'aliment du lot HP était riche en matière azotée peu dégradable dans le rumen, estimée par les PDIA (110 g de PDIA $/ \mathrm{kg}$ de MS) et l'aliment BP était pauvre en PDIA (38 g de PDIA/kg de MS).

Comme il n'existe aucune interaction entre les effets de la date de mise à l'herbe et de la nature des matières azotées du concentré, le tableau 9 présente les résultats moyens de l'effet de la nature des matières azotées quelle que soit la date de mise à l'herbe. L'apport d'un concentré riche en matière azotée peu dégradable dans le rumen chez les chèvres au début du pâturage de printemps, améliore significativement la production laitière alors que les taux butyreux et protéique ne sont pas significativement modifiés.

En comparant les résultats des expériences 4, 6, 8 et 9 concernant les caractéristiques énergétiques et azotées de l'aliment concentré distribué, il semble que la composition de l'aliment concentré peut avoir une influence sur les performances laitières des chèvres au pâturage. Les caractéristiques azotées de l'aliment concentré influenceraient nettement plus la production laitière que les caractéristiques énergétiques. En effet, l'augmentation de la teneur azotée

Tableau 9. Effet de la teneur de l'aliment concentré en protéines peu dégradables dans le rumen sur les performances des chèvres laitières au pâturage (expérience 4).

\begin{tabular}{|l|c|c|}
\hline \multicolumn{1}{|c|}{ Lot $^{*}$} & BP & HP \\
\hline Teneur en PDIA de l'aliment concentré (g/kg MS) & 38 & 110 \\
\hline Production de lait (kg/j/chèvre) & $3,71^{\mathrm{a}}(0,90)^{* *}$ & $4,08^{\mathrm{b}}(1,05)$ \\
\hline Taux butyreux (g/kg lait) & $36,8(4,29)$ & $35,3(4,50)$ \\
\hline Taux protéique (g/kg lait) & $31,3(2,40)$ & $31,6(2,32)$ \\
\hline
\end{tabular}

* Lot BP : chèvres recevant un aliment concentré pauvre en protéines peu dégradables dans le rumen.

Lot HP : chèvres recevant un aliment concentré riche en protéines peu dégradables dans le rumen.

** L'écart-type est placé entre parenthèses.

Entre les lots, les valeurs affectées des lettres a et b sont significativement différentes au seuil $P<0,05$. 
de l'aliment concentré et de sa proportion de matière azotée peu dégradable dans le rumen augmente la production laitière des chèvres au pâturage, notamment au début de la saison. En revanche, les caractéristiques azotées n'ont pas d'effet significatif sur le taux butyreux alors que l'apport d'un aliment concentré riche en fibres, en matière grasse et pauvre en amidon l'améliore nettement. Quant au taux protéique, il n'est influencé ni par les caractéristiques énergétiques ni par les caractéristiques azotées de l'aliment concentré.

\section{8 / Discussion et conclusion}

Les 9 expériences rapportées ici ont permis de montrer que des productions laitières élevées de chèvres primipares (700 à $850 \mathrm{~kg}$ de lait par an) et multipares $(900$ à $1150 \mathrm{~kg}$ de lait par an et plus de $5 \mathrm{~kg}$ de lait par jour et par chèvre au pic de lactation) peuvent être obtenues dans un système d'alimentation cherchant à optimiser l'utilisation d'herbe pâturée en couvrant environ de 50 à $75 \%$ des besoins énergétiques des chèvres par l'herbe et en ne distribuant pas plus de $0,8 \mathrm{~kg}$ d'aliments concentrés par jour et par chèvre. De tels résultats obtenus sur chèvres laitières dans des conditions d'élevage intensif sont très proches des résultats enregistrés sur vaches dans des conditions comparables (Peyraud et al 1995, Delaby et al 2003).

Les questions essentielles à se poser, à la vue des résultats du Pradel, sont d'une part, leur aptitude à être généralisés et d'autre part, la stratégie à adopter pour obtenir des performances laitières comparables dans des conditions d'élevage différentes. Les expériences du Pradel ont été conduites dans des conditions favorables à la pousse de l'herbe en climat méditerranéen humide avec plus d'un mètre d'eau par an. Elles ont fait avancer les connaissances sur des techniques de pâturage à appliquer aux caprins qui peuvent être transférables à d'autres conditions environnementales.

Ces expériences, en particulier l'expérience 5 , ont permis aussi de préciser que la qualité de l'herbe est un facteur plus important que la quantité de concentré distribué. En effet, des quantités différentes d'aliments concentrés modifient assez peu la production et la composition du lait si les chèvres ont la possibilité d'ajuster leur ingestion d'herbe, c'est-à-dire si l'herbe disponible au pâturage est suffisante, ce qui est le cas au Pradel avec $3 \mathrm{~kg} / \mathrm{j}$ de MS offerte par chèvre, si l'herbe est de bonne qualité et si les chèvres restent assez de temps au pâturage pour pâturer une quantité d'herbe suffisante. Au cours de ces neuf expériences et dans les conditions d'élevage de la région, il a été constaté qu'une durée d'accès journalier au pâturage supérieure à 11 heures par jour n'améliorait pas en général le niveau d'ingestion par rapport à une durée d'accès de 10 à 11 heures au pâturage. Avec une durée de moins de 10 heures d'accès au pâturage, le niveau d'ingestion diminue rapidement. Si elle est réduite à $7-8$ heures, le niveau d'ingestion n'est déjà plus que de $50-70 \%$ de l'ingestion maximale. Il est intéressant de noter que des durées d'accès au pâturage de 10 - 11 heures ne sont pas encore très fréquentes en France car cela demande une remise au pâturage après la traite du soir.

Les expériences du Pradel ont aussi montré que le pâturage continu permet d'obtenir des performances laitières comparables à celles obtenues avec du pâturage tournant. En revanche, en ce qui concerne le pâturage tournant, il permet de récolter un peu plus d'excédents sous forme de foin $(20 \%$ environ). Cet élément est à prendre en compte par rapport à l'autonomie fourragère. Dans les deux cas et en particulier en pâturage continu, la gestion du pâturage doit être précise en fonction de la quantité et de la qualité d'herbe disponible et du comportement des animaux. Elle est plus facile et à un coût de main-d'œuvre plus faible dans le cas du pâturage continu. En revanche, d'après ce qui a été observé au Pradel, la technique du pâturage tournant semble plus indiquée dans le cas de légumineuses.

Au Pradel, une mise à l'herbe précoce (début Mars) s'est révélée plus favorable à une production laitière optimale qu'une mise à l'herbe plus tardive (un mois après). Dans le cas d'une mise à l'herbe précoce, la transition d'un régime de foin à un régime d'herbe verte est plus longue et doit être réalisée à l'aide de contrôles systématiques quotidiens. La maîtrise de la pousse de l'herbe étant un point-clé pour garder une qualité d'herbe optimale, il est plus facile avec une sortie précoce des animaux de gérer les moments de fortes pousses, principalement du fait de cette transition progressive et de la capacité des chèvres à ingérer de grandes quantités d'herbe lors de fortes disponibilités fourragères. Par ailleurs, l'utilisation précoce des parcelles a aussi pour conséquence de retarder la date de fauche et coïncide souvent avec des périodes où les conditions climatiques sont plus favorables à la fauche. Il est probable que dans d'autres conditions de pâturage, une mise à l'herbe précoce garde son intérêt même si les dates de mise à l'herbe doivent être ajustées à chaque condition agroclimatique particulière.

Le niveau et la nature des sources azotées de l'aliment concentré ont tendance à plus influencer la production laitière que le niveau et la nature des sources énergétiques du concentré, en particulier au printemps. Ce résultat s'expliquerait par la teneur relativement élevée de l'azote dégradable de l'herbe à cette époque. En conséquence, l'apport de sources azotées peu dégradables rééquilibrerait les différentes sources azotées ingérées et serait dans ce cas plus favorable à une production de lait élevée comme Hadjipanayiotou et Morand-Fehr (1991) l'avaient déjà indiqué dans des conditions alimentaires variées. Ce résultat étant assez général, il pourrait s'observer aussi dans des conditions différentes de celles du Pradel.

Les expériences du Pradel ont abordé des questions sur le pâturage des caprins qui jusqu'ici n'avaient été que très peu ou pas étudiées surtout avec des chèvres laitières présentant un potentiel laitier élevé. Ils ont donc complété les résultats plus ponctuels obtenus par Jagusch et al (1981) et Soryal et al (2004) avec des chèvres laitières moins productives.

Par ailleurs, il est difficilement compréhensible, à la lumière des résultats du Pradel, que la chèvre ait encore la réputation de mal exploiter le pâturage. Celle-ci s'est développée à partir de constatations de terrain ou d'anciens résultats expérimentaux (Morand-Fehr 1981, de Simiane et al 1981, Masson et de Simiane 1981). Il faut reconnaître qu'à l'époque de ces observations et de ces travaux expérimentaux, les caractéristiques du comportement des caprins étaient moins bien connues, en particulier leur choix sélectif très développé, face à un fourrage ou une ration dès que la quantité est proche de couvrir leurs besoins (Bordi et al 1994, Morand-Fehr 2003). Les observations du Pradel, parfois difficiles à quantifier, montrent aussi qu'une gestion rigoureuse et précise du pâturage par un contrôle quotidien de l'éleveur est absolument nécessaire pour tenir compte de ce comportement et pour éviter le gaspillage d'une partie du couvert végétal. Les meilleures performances des chèvres obtenues avec une mise à l'herbe précoce par rapport à une mise à l'herbe plus tardive s'expliquent principalement par un gaspillage d'herbe beaucoup plus réduit. De plus, la plupart des expériences anciennes sur le pâturage des caprins utilisait des chèvres peu habituées au pâturage, en particulier au cours de leur croissance (Masson et de Simiane 1981, de Simiane et al 1981, Morand-Fehr et al 1991) alors qu'au 
Pradel, les chevrettes destinées au renouvellement du troupeau laitier sortent au pâturage pendant leur période de croissance (Lefrileux et Van Quackebeke 1996). Ce point est essentiel pour obtenir de bonnes performances laitières avec des systèmes d'alimentation à base de pâturage, en particulier pour préparer les chèvres à avoir une capacité d'ingestion élevée, liée au volume de leur rumen pendant leur vie productive (MorandFehr et Sauvant 1987).

Enfin, il faut appliquer une stratégie de conduite de l'alimentation adaptée, en particulier ne pas hésiter à éliminer la distribution d'un autre fourrage à l'auge et limiter l'apport d'aliments concentrés quand la quantité et la qualité de l'herbe sont suffisantes; ce que Jagusch et al (1981) avaient déjà préconisé avec des chèvres à potentiel laitier sensiblement moins élevé. C'est d'ailleurs ce qui est également préconisé pour les vaches laitières (Delaby et al 2003). En revanche, il faut augmenter les apports complémentaires dès que l'herbe devient insuffisante comme en été, ou de médiocre qualité selon les conditions climatiques, comme cela se pratique déjà dans les élevages caprins en France (Foubert et Jénot 2005).
A partir des observations quantifiées ou non quantifiées pendant ces expériences, il apparaît que pour une production intensive de lait de chèvre, le pâturage est plus complexe à mettre en œuvre sur le plan technique qu'une alimentation à l'auge, probablement plus que pour d'autres ruminants comme le pensent certains éleveurs caprins. Le rôle de l'éleveur est donc essentiel pour observer l'état des parcelles, leur évolution plus ou moins rapide et le comportement des chèvres du troupeau. En conséquence, pour que les expériences de chacun profitent aux autres et compte tenu de la faible information disponible sur le sujet, une organisation en réseau des éleveurs pour s'échanger leurs expériences et leurs observations personnelles est souhaitable et a montré son efficacité dans la région.

$\mathrm{Au}$ moment où le consommateur devient plus exigeant vis-à-vis de la qualité des produits et du bien-être des animaux d'élevage, l'utilisation du pâturage par la chèvre laitière est susceptible de se développer à l'avenir en tenant moins compte de la réputation de la chèvre de mal valoriser le pâturage. En outre, il est probable que comme chez la vache (Bugaud et al 2002), les caracté- ristiques du pâturage influencent la flaveur et la texture des fromages chez une espèce autant fromagère que l'espèce caprine. En tout cas, il faut espérer que les résultats présentés dans cet article contribuent à dissiper les réticences présentes encore chez certains éleveurs pour adopter des systèmes d'alimentation à base de pâturage.

Toutefois, des études restent à développer, en particulier sur les niveaux d'ingestion d'herbe au pâturage - notamment avec des prairies multi-espèces afin d'établir les lois de substitution entre l'herbe et le concentré, sur le chargement des chèvres au pâturage selon la masse fourragère disponible et sur les traitements à réaliser en vue de bien maîtriser le parasitisme selon la gestion du pâturage et les conditions agroclimatiques. Même si l'objet du présent article n'était pas le parasitisme des chèvres au pâturage, sa maîtrise est essentielle pour que les systèmes d'alimentation à base de pâturage soient efficaces. Nous renvoyons le lecteur à l'article de Hoste et al (2012) dans lequel les connaissances actuelles sur le parasitisme des chèvres au pâturage sont rapportées.

\section{Références}

Barillet F., Astruc J.M., Clément V., Lagriffoul G., Marie-Etanulin C., Piacère A., Rupp R., Manfredi E., 2004. Improving milk yield and composition in dairy sheep and goats through genetics. Proc. Int. Symp. of the future of the sheep and goats dairy sector, Zaragoza, Espagne, 3, 134-142.

Bordi A., de Rosa G., Napolitano F., Vesce G., Rubino R., 1994. Influence of feeding supplementation on goat grazing behaviour. Cahiers Options Méditerranéennes, 5, 57-68.

Bugeaud C., Buchin S., Hauwuy A., Coulon J.B., 2002. Texture et flaveur du fromage selon la nature de pâturage : cas du fromage d'Abondance. INRA Prod. Anim., 15, 31-36.

Chilliard Y., Ferlay A., Rouel J., Lamberet G., 2003. A review of nutritional and physiological factors affecting goat milk lipid synthesis and lipolysis. J. Dairy Sci., 86, 17511770.

Chilliard Y., Glasser F., Enjalbert F., Ferlay A., Bocquier F., Schmidely P., 2007. Données récentes sur les effets de l'alimentation sur la composition en acides gras du lait de vache, de chèvre et de brebis. Renc. Rech. Rum., 14, 321-328.

Coulon J.B., Rémond B., 1991. Variation in milk output and milk protein content response to the level of energy supply to the dairy cows: a review. Livest. Prod. Sci., 29, 31-47.

Delaby L., Peyraud J.L., 1994. Effect of the energy source in the concentrate on the performance of grazing dairy cows. Renc. Rech. Rum., 1, 113-116.
Delaby L., Peyraud J.L., Delagarde R., 2003. Faut-il complémenter les vaches laitières au pâturage ? INRA Prod. Anim., 16, 183-195.

Fedele V., Pizzillo M., Claps S., MorandFehr P., Rubino R., 1993. Grazing behaviour and diet selection of goats on native pasture in Southern Italy. Small Rum. Res., 11, 305-322.

Fedele V., Rubino R., Claps S., 1997. Forage production, herbage utilization and productive performances of goats reared using different feeding systems. Rivista di Agronomia, 31, 331-336.

Fedele V., Pizzoferrato L., Manzi P., Cifuni G.F., Sepe L., Di Napoli M.A., 2007. Grazed or preserved forage: a global evaluation of goat milk and cheese quality. In: The quality of goat products: models and tools for evaluation and promotion, Rubino R., Sepe L. (Eds), Impronta Digitale Publ., Italya, 195-198.

Foubert C., Jénot F., 2005. Indoors complementary feeds supplies for grazing dairy goats in Poitou-Charentes. Renc. Rech. Rum., 12, 251.

Gautier D., Moulin C.H., 2004. Intérêt du pâturage hivernal sur parcours pour les exploitations ovines : exemple des Préalpes du Sud. INRA Prod. Anim., 17, 275-286.

Hadjipanayiotou M., Morand-Fehr P., 1991. Intensive feeding of dairy goats. In: Goat Nutrition. Morand-Fehr P. (Ed), Pudoc, Wageningen, Pays-Bas, 197-208.

Hervieu J., Morand-Fehr P., 1999. Comment noter l'état corporel des chèvres ? Réussir, La Chèvre, 231, 26-32.
Hoste H., Ehrhardt N., Paraud C., Rieux A., Mercier P., Valas S., Andreoletti O., Corbière F., Schelcher F., Lacroux C., de Crémoux R., Alvinerie M., Chartier C., 2012. Recherches en pathologie caprine : applications et perspectives. In : Elevage caprin. Baumont R., Sauvant D. (Eds). Dossier, INRA Prod. Anim., 25, 277290.

INRA, 2007. Alimentation des bovins, ovins et caprins. Besoins des animaux. Valeurs des aliments. Editions Quae, Paris, France, 186-198.

Jagusch K.T., Kidd G.T., Lynch R., 1981. Commencing a dairy goat enterprise based on the grazing or rye-grass-white clover pasture. Symp. Int. Nutr. Syst. Goat Feeding, Tours, France, 1, 383-391.

Lefrileux Y., Van Quackebeke E., 1996. Alimentation des chevrettes d'élevage sur pâturage de luzerne. Renc. Rech. Rum., 3, 256.

Lefrileux Y., Le Mens P., Guérin B., 2000. Influence de la mise à l'herbe et de la nature azotée du concentré sur la transformation fromagère. J. Nat. Tech., Fromagers, Oraison, 7.

Lefrileux Y., Le Scouarnec J., Pommaret A., Cirier N., 2005. Effects of concentrate supply with commercial feed or maize on milk performances and sensorial quality of milk in grazing dairy goats. Renc. Rech. Rum., 12, 250.

Lefrileux Y., Morand-Fehr P., Pommaret A., 2008. Capacity of high milk yielding goats for utilizing cultivated pasture. Small Rum. Res., 77, 113-126. 
Ligios S., Sitzia M., Fois N., Decandia M. Molle G., Roggero P.P., Casu S., 2002. Effect of grass availability and nature on ingestion and milk performances in grazing ewes. Options Méditerranéennes Série B, 42, 73-84.

Min B.R., Hart S.P., Sahlu T., Satter L.D., 2005. The effect of diets on milk production and composition, and on lactation curves in pastured dairy goats. J. Dairy Sci., 88, 26042615.

Masson C., de Simiane M., 1981. Level of intake in grazing dairy goats. Symp. Int. Nutr. Syst. Goat Feeding, Tours, France, 1, 357-361.

Masson C., Hacala S., 1985. Utilisation du ray-grass d'Italie en pâturage rationné par la chèvre laitière. Fourrages, 101, 31-35.

Morand-Fehr P., 1981. Behavioral and digestive characteristics in goats. Symp. Int. Nutr. Syst. Goat Feeding, Tours, France, 1, 2145 .

Morand-Fehr P., 2003. Dietary choices of goats at the trough. Small Rum. Res., 49, 231239.

Morand-Fehr P., 2005. Recent developments in goat nutrition and application: a review. Small Rum. Res., 60, 25-43.

Morand-Fehr P., Sauvant D., 1987. Feeding strategies in goats. $6^{\text {th }}$ Int. Conf. on Goats, Brasilia, Brésil, 1275-1303.

Morand-Fehr P., Sauvant D., 1989. Goats. In: Ruminant Nutrition: recommended allowances and feed tables. Jarrige R. (Ed), John Libbey, London, UK, 169-179.

Morand-Fehr P., Bas P., Blanchart R., Daccord R., Giger-Reverdin S., Gihad E.A.,
Hadjipanayiotou M., Mowlem A., Remeuf F., Sauvant D., 1991. Influence of feeding on goat milk composition and technological characteristics. In: Goat Nutrition. Morand-Fehr P., (Ed), Pudoc, Wageningen, Pays-Bas, 209-224.

Morand-Fehr P., Fedele V., Decandia M. Lefrileux Y., 2007. Influence of farming and feeding systems on composition and quality of goat and sheep milk. Small Rum. Res., 68, 20-34.

Munger A., Jans F., 2001. Rotational versus continuous grazing with dairy cows and type of supplementation effects. Grassland Sci. Eur., 7 , 254-255.

Peyraud J.L, Delagarde R., Delaby L., 1995. Influence des conditions d'exploitation du pâturage et des caractéristiques animales sur les quantités ingérées par les vaches laitières : analyse et prédiction. Renc. Rech. Rum., 2, 37-44.

Peyraud J.L., Delaby L., Delagarde R., Marquis B., 1998. Effet de l'apport de concentré énergétique et de quantités d'herbe offertes sur l'ingestion de vaches laitières au pâturage. Renc. Rech. Rum., 5, 217-221.

Pulido R.G., Leaver J.D., 2003. Continuous and rotational grazing in dairy cows. The interactions of grazing system with level of milk yield, sward height and concentrate level. Grass and Forage Sci., 58, 265-275.

Sayers H.J., Mayne C.S., 2001. Effect of early turn out to grass in spring on dairy cow performance. Grass and Forage Sci., 56, 259-267.

Schmidely P., Morand-Fehr P., Sauvant D., 2005. Influence of extruded soybeans with or without bicarbonate on milk performance and fatty acid composition of goat milk. J. Dairy Sci., 88, 757-765.
Simiane (de) M., Giger S., Blanchart G., Huguet L., 1981. Nutritional value and utilization of intensively cultivated roughages. Symp. Int. Nutr. Syst. Goat Feeding, Tours, France, 1, 274-298.

Sitzia M., Roggero P.P., Fois N., Molle G., 1997. Grazing management of an Italian ryegrass sward with dairy sheep in the Mediterranean environment. Proc. 18 ${ }^{\text {th }}$ Int. Grassland Congr., Winnipeg, Canada, 319-323.

Soryal K.A., Zeng S.S., Min B.R, Hart S.P., Beyene F.A., 2004. Effect of feeding systems on composition of goat milk and yield of Doniati cheese. Small Rum. Res., 54, 121-129.

Van Quackebeke E., Lefrileux Y., Pommaret A., Audic C., 1996a. Comparaison de deux systèmes d'alimentation des chèvres laitières. Renc. Rech. Rum., 3, 289-292.

Van Quackebeke E., Lefrileux Y., Pommaret A., Borgida L.P., 1996b. Effet du niveau de complémentation énergétique et azotée sur la production laitière des chèvres alimentées en lot. Renc. Rech. Rum., 3, 118.

Van Quackebeke E., Lefrileux Y., Pommaret A., 1997. Comparaison de deux modes de pâturage des chèvres laitières : pâturage continu pâturage tournant. Renc. Rech. Rum., 4, 154.

Wilson L.A., Roberts D.J., Henderson A.R., 2002. Performance of grazing dairy cow fed different concentrate energy types and an additive to reduce protein degradability. Proc. $19^{\text {th }}$ Gen. Meeting Eur. Grassland Fed., La Rochelle, France, 268-269.

\section{Résumé}

Neuf expériences ont été réalisées à la station expérimentale du Pradel (Sud-Est de la France) de 1991 à 2000 sur la conduite au pâturage des chèvres laitières hautes productrices $(800-1100 \mathrm{~kg}$ de lait/chèvre/an). Elles portaient sur le type de pâturage à adopter (pâturage tournant ou continu), les conditions de la mise à l'herbe et la stratégie de complémentation afin d'évaluer leurs effets sur les performances laitières (production et composition du lait). Deux expériences comparant pendant 3 ans et 100 jours, respectivement deux systèmes d'alimentation, en chèvrerie ou au pâturage, ont montré que le pâturage couvre plus de $50 \%$ des besoins énergétiques des chèvres et que les productions laitières et les taux butyreux et protéique ne sont jamais significativement différents entre les deux conduites. Dans une troisième expérience aucune différence significative n'a été observée sur la production et la composition du lait de chèvres conduites pendant $\mathbf{1 0 0}$ jours au printemps en pâturage tournant ou en pâturage continu à surface identique et sur des couverts végétaux comparables. Une quatrième expérience de 120 jours a permis de montrer qu'une mise à l'herbe précoce (début Mars) permet une meilleure production laitière qu'une mise à l'herbe plus tardive, vers le 31 Mars. Quatre expériences d'une durée de 12 à 16 semaines ont comparé différents niveaux de complémentation des chèvres au pâturage. L'augmentation de l'apport d'aliment concentré améliore de façon limitée la production laitière, en particulier au dessus de $0,8 \mathrm{~kg}$ de concentré/jour/chèvre. Avec de l'herbe de très bonne valeur alimentaire, cette augmentation est de $10 \mathrm{~g}$ de lait/100 $\mathrm{g}$ de concentré ajouté alors qu'elle est de $40 \mathrm{~g}$ avec de l'herbe à un stade plus avancé. La composition du lait est très peu modifiée par l'apport de concentré. Une dernière expérience de 100 jours et certaines expériences précédentes ont précisé les effets de la composition de l'aliment concentré. La nature des sources azotées (protéines peu ou fortement dégradables dans le rumen) influence plus la production laitière que celle des sources énergétiques (amidon vs fibres et matière grasse). Ainsi une production de lait de 1000-1100 kg par an peut être obtenue avec un système alimentaire où le pâturage couvre une part importante des besoins énergétiques totaux des chèvres (50 à $75 \%$ environ). Pour cela il est nécessaire que le potentiel génétique des chèvres le permette, que la durée journalière de pâturage soit suffisante (10-11 heures par jour), que les apports de fourrages distribués à l'auge soient très limités ou supprimés, que ceux d'aliments concentrés soient limités à environ $0,8 \mathrm{~kg} / \mathrm{chèv} r$ /jour et surtout qu'une gestion du pâturage basée sur des contrôles journaliers et des observations précises soit mise en place avec rigueur par l'éleveur.

\section{Abstract}

\section{Capacity of high milk yielding goats to utilize cultivated pastures}

Nine experiments were carried out at the experimental station of Le Pradel (south-eastern France) from 1991 to 2000 on the management of grazing high milk yielding goats $(800-1100 \mathrm{~kg}$ milk/goat/year) to compare continuous vs rotational grazing, the early or late turn out and different managements of concentrate supplies so as to get optimal milk production and composition. Two experiments 
comparing during 3 years and 100 days two feeding systems, indoors exclusively or based on grazing, showed that grazing covered more than $50 \%$ of energy requirements of goats and that the milk production and fat and protein percentages were not significantly different between the two feeding systems. In a third experiment no significant difference in milk production and composition was observed between goats managed on rotational or continuous grazing. A fourth experiment showed that a better milk production and composition was achieved with an early turn out (around 1st March) in comparison with a later turn out around 31 March. Four experiments lasting 12 - 16 weeks compared various levels of concentrate supplies in grazing goats. The higher concentrate supply increased milk production in a limited way, particularly above $0.8 \mathrm{~kg}$ of concentrate/day/goat and with grass of high dietary value (10 g milk per $100 \mathrm{~g}$ concentrate vs $40 \mathrm{~g}$ concentrate with lower quality grass). Milk composition was very little influenced. A last experiment and several previous experiments investigated the effects of the concentrate composition. The nature of nitrogen sources (highly or little degradable protein in the rumen) more strongly influenced goat milk production than the type of energy resources (starch vs fibre and fat). Thus an annual production around $1000-1100 \mathrm{~kg}$ of milk in multiparous dairy goats can be obtained with a feeding system based on cultivated grazing which covers the main part of energy requirements (from around 50 to $75 \%$ ). For that, the genetic potential of goats has to be sufficient, the daily grazing duration has to reach 10 to $11 \mathrm{~h} / \mathrm{day}$, the supplies of conserved fodders have to be very limited or withdrawn, the concentrate supplies have to be limited to around $0.8 \mathrm{~kg} / \mathrm{goat} / \mathrm{day}$, and the pastures and animal behaviour on grazing need to be managed by goat farmers with daily accurate observations and controls.

LEFRILEUX Y., MORAND-FEHR P., POMMARET A., 2012. Aptitude des chèvres hautes productrices de lait à valoriser les prairies temporaires au pâturage. In : Elevage caprin. Baumont R., Sauvant B. (Eds). Dossier, INRA Prod. Anim., 25, $277-290$. 